\title{
STATIONARY MULTIPLE SPOTS FOR REACTION-DIFFUSION SYSTEMS
}

\author{
JUNCHENG WEI AND MATTHIAS WINTER
}

\begin{abstract}
In this paper, we review analytical methods for a rigorous study of the existence and stability of stationary, multiple spots for reaction-diffusion systems. We will consider two classes of reactiondiffusion systems: activator-inhibitor systems (such as the Gierer-Meinhardt system) and activatorsubstrate systems (such as the Gray-Scott system or the Schnakenberg model).

The main ideas are presented in the context of the Schnakenberg model, and these results are new to the literature.

We will consider the systems in a two-dimensional, bounded and smooth domain for small diffusion constant of the activator.

Existence of multi-spots is proved using tools from nonlinear functional analysis such as LiapunovSchmidt reduction and fixed-point theorems. The amplitudes and positions of spots follow from this analysis.

Stability is shown in two parts, for eigenvalues of order one and eigenvalues converging to zero, respectively. Eigenvalues of order one are studied by deriving their leading-order asymptotic behavior and reducing the eigenvalue problem to a nonlocal eigenvalue problem (NLEP). A study of the NLEP reveals a condition for the maximal number of stable spots.

Eigenvalues converging to zero are investigated using a projection similar to Liapunov-Schmidt reduction and conditions on the positions for stable spots are derived. The Green's function of the Laplacian plays a central role in the analysis.

The results are interpreted in the biological, chemical and ecological contexts. They are confirmed by numerical simulations.
\end{abstract}

\section{Introduction: An Overview}

One of the central issues in developmental biology is to understand how spatial patterns arise as an embryo develops from a single fertilized cell into an adult. In 1952, Alan Turing [64] proposed that a system of interacting chemicals could be driven unstable by diffusion and evolve into a spatial pattern. This was an example of self-organization giving rise to emergent behavior. Since his seminal work, many models have been proposed, from a huge spectrum of applications. A good account of these models can be found in J. Murray's book [43]. Among those models, in particular three stand out: the GiererMeinhardt system [17] in biological development, the Gray-Scott model and the Schnakenberg model in chemical reaction theory. In a two-dimensional domain, intricate spatially localized patterns, consisting of either spots, stripes, mixed spot-stripe patterns, or space-filling curves, have been observed in numerical simulations of those Turing systems. For activator-inhibitor systems, such as the well-known Gierer-Meinhardt model of biological morphogenesis, spot and stripe patterns are ubiquitous ([17], [41], [42], [34], [35]). For the Gray-Scott or Schnakenberg model of theoretical chemistry, an even greater diversity of spatio-temporal patterns occur, including spot-replication behavior, spatio-temporal chaos of spot patterns and labyrinthine patterns of stripes ([31], [32], [50], [51], [52]).

1991 Mathematics Subject Classification. Primary 92C15, 35K45; Secondary 35J55.

Key words and phrases. Pattern formation, Multiple spots, Reaction-diffusion systems. 
Most of the previous analysis of the stability of localized patterns has been based on a weakly nonlinear theory, where the solution is assumed to be close to some spatially uniform state across the domain. However, numerical and analytical analysis shows that the stable patterns are far from the uniform state. In this paper, we shall give a unified and rigorous treatment of multiple spots which can occur for several types of two-component, singularly perturbed reaction-diffusion systems in a bounded, two-dimensional domain.

We begin with a classification of reaction-diffusion systems.

1.1. Reaction-Diffusion Systems. A two-component reaction-diffusion system in $R^{2}$ is given by

$$
v_{t}=\epsilon^{2} \Delta v+f(u, v), \quad \tau u_{t}=D \Delta u+g(u, v) .
$$

The unknowns $u=u(x, t)$ and $v=v(x, t)$ represent the concentrations of two chemicals at a point $x \in \Omega \subset R^{2}$ and at a time $t>0$, respectively, where $\Omega$ is a bounded and smooth domain in $R^{2}$; $\Delta:=\sum_{j=1}^{2} \frac{\partial^{2}}{\partial x_{j}^{2}}$ is the Laplace operator in $R^{2} ; \nu(x)$ is the outer normal at $x \in \partial \Omega ; \epsilon^{2}$ and $D$ are positive diffusion constants; $\tau \geq 0$ is a non-negative time relaxation constant. Note that for the special case $\tau=0$ we have a mixed parabolic-elliptic system, otherwise a parabolic system. The functions $f(u, v)$ and $g(u, v)$ describe nonlinear reaction terms which are explained in more detail below.

It is well-known that for two-component reaction-diffusion systems the Turing instability, which leads to pattern formation [64], is possible for exactly two types of systems which are characterized by the signs of the Jacobian at a homogeneous, positive, steady state [43]. After a suitable relabelling of the two components these two types can be written as follows:

$$
J_{1}=\left[\begin{array}{cc}
+ & - \\
+ & -
\end{array}\right], \quad J_{2}=\left[\begin{array}{cc}
+ & + \\
- & -
\end{array}\right] .
$$

We now consider some important examples of reaction-diffusion systems for both types.

Activator-inhibitor systems which are suggested in equation (12) of [17] to have the reaction terms

$$
f(u, v)=-v+\frac{v^{p}}{u^{q}}, \quad g(u, v)=-u+\frac{v^{r}}{u^{s}}, \quad \text { where } 1<\frac{q r}{(p-1)(s+1)}, 1<p
$$

(after re-scaling) are of type one. It is commonly assumed that there is a fast-diffusing inhibitor, $u$, which inhibits the production of a slowly-diffusing activator, $v$. On the other hand, $v$ activates itself and the inhibitor. This mechanism drives sharply localized spatial spots of activator coupled with nearby shallow peaks of inhibitor. A particular case of activator-inhibitor system which is now commonly called the Gierer-Meinhardt system is the special case $(p, q, r, s)=(2,1,2,0)$.

Activator-substrate systems which are suggested in equation (11) of [17] (called the Depletion Model there) to have the reaction terms

$$
f(u, v)=-v+u v^{2}, \quad g(u, v)=A-\mu u-u v^{2}, \quad \text { where } A>0
$$

(after re-scaling) are of type two. It is commonly assumed that there is a fast-diffusing substrate, $u$, which is consumed by a slowly-diffusing activator, $v$, and supplied to the system at a constant 
rate. On the other hand, $v$ activates itself. This mechanism drives sharply localized spatial spots of activator coupled with nearby shallow dips of substrate.

Further, to get pattern formation, for activator-substrate systems the feed-rate must be large enough: if the feed-rate is too small there is not enough substrate to support the production of activator and any pattern will eventually die out. This is in contrast to the activator-inhibitor case, which does not have any feeding mechanism.

The effect of the feed-rate is modelled by the constant $A$. To get stable multi-spots one has to assume that $A$ is large enough (see Theorem 2.2 below).

Particular cases of activator-substrate systems are $\mu=1$ which is now commonly called the GrayScott system [18], [19] and $\mu=0$ which is now commonly called the Schnakenberg model [59]. In both cases the reaction kinetics is derived from simple chemical reactions using the mass balance law.

So there is a marked difference between activator-inhibitor and activator-substrate systems: Near activator peaks the inhibitor has high values, but the substrate has low values. This is easy to understand intuitively, as in the first case high values of activator lead to strong activation of inhibitor, resulting in an inhibitor peak, whereas in the second case high values of activator lead to fast consumption of substrate, causing a substrate dip. This difference is clearly reflected in our analytical results and numerical simulations for multi-spot patterns.

1.2. Previous Results on Peaked Solutions. Let us now give an overview of the literature to the problem. For the one-dimensional Gierer-Meinhardt model we refer to [7], [63]. In [7] it has been shown that a general two-component, singularly perturbed system that exhibits large-amplitude pulse patterns has a leading order 'normal form' which is given by (1.1). For the two-dimensional Gierer-Meinhardt system existence and stability of multi-spots have been analyzed in [73], [74] [75] (proving a conjecture of Ni [44]). For the limit $D \rightarrow \infty$ (shadow system) see [68].

For the Gray-Scott system we refer to [54] (numerical simulation), [41], [42] (asymptotic expansion), [8], [9], [10] (rigorous proofs for one dimension), [69], [70], [76], [77] (rigorous study of multi-spots for higher dimensions), [7], [45], [46], [48], [49], [25], [26], [62] (rigorous study of instability mechanisms of multi-spots).

The Turing bifurcations for the Schnakenberg model with spatially varying diffusion coefficients are studied on a spatial two-dimensional square in [1]. It is shown how this spatial variation can be used to partially reduce the degeneracy in the Turing bifurcation. Interesting phenomena are established which include stable subcritical striped patterns and stripes losing stability super-critically to give stable spotted patterns.

For the Schnakenberg model on a one-dimensional interval the existence and stability of multiple interior spike solutions have been established in the symmetric case (i.e. spots of equal amplitudes) [22] and in the asymmetric case (i.e. spots of two different amplitudes) [71]. 
The motion of spots has been analyzed in [12], [45]. For absolute instability see [58]. For chaotic behavior see [50]. Singular eigenvalue problems for reaction-diffusion equations have been studied in [47].

Reviews on pattern formation for reaction-diffusion systems and its biological, chemical and ecological implications are given in [33], [38], [37] [67]. For an overview of biological modelling we refer to $[43]$.

1.3. Biological implications. We now discuss biological, chemical and ecological implications of these models.

Biological applications of the Gierer-Meinhardt model and its generalizations to such diverse biological processes as animal skin patterns, patterns on tropical sea shells, organ formation, nerve cell and brain activity, regeneration in hydra and segmentation have been described in [38], [39].

A Turing model has been suggested to explain the development of pigmentation patterns on certain species of growing angle-fish such as Pomacanthus semicirculantus where colored stripes are observed which change their number, size and orientation [27]. After this model was refined, adding effects such as cell growth and movement, also stripes of various thickness could be explained [53].

For reaction-diffusion systems on growing domains, which is a good model for the growth of organisms, we mention [3], [4], [34], [35].

Recently in [61] hair follicle arrangements in mice have been modelled by a reaction-diffusion system, where the WNT and DKK proteins serve as an activator and inhibitor, respectively, and experiments are combined with numerical computations. See also the perspective in [36].

In chemistry, open systems in which chemicals are fed into the system play an important role. Simple models for this are Gray-Scott and Schnakenberg [65].

The Gray-Scott model and its relevance as a model for the ferrocyanide-iodate-sulfate (FIS) reaction have been investigated both numerically and experimentally in [31], [32]. Self-replication spots have been investigated numerically and by formal analysis in [56], [57].

In chemistry one criticism has been that the diffusion constants of the different chemical substances are probably not very different. It has been shown mathematically, using Turing instability, that chemical patterns with equal diffusion coefficients are possible [65]. These ideas have then been applied to the Belousov-Zhabotinskii reaction [55]. However, a mathematical analysis of multi-spots in one space-dimension for the Gray-Scott system revealed that they are unstable for equal diffusion constants [14], [15], [20], [21].

For the CIMA reaction experimentally found pattern formation [2], [6], [51], [52] could successfully be explained by reaction-diffusion modelling [30].

Even though the Schnakenberg model is unquestionably a simplification of processes in chemical reactors many of the patterns observed experimentally can be computed with the Schnakenberg model, such as multi-spots forming hexagonal arrays, stripes and wiggled stripes [11]. 
Let us finally comment on ecology. In [33] it is argued that pattern and scale are the central processes in ecology which unify population biology and ecosystems science and are essential for pure and applied ecology. The main task is therefore to understand the mechanism leading to pattern formation which acts on various scales. These patterns can be explained by Turing instabilities [60] and Turing patterns have the advantages that no genetic information is required and that almost every conceivable pattern can be explained. Patterns are important in ecology as they can explain diversity. So the study of Turing patterns in an ecological context is very interesting and important.

1.4. Analysis of Multi-spots. We now turn to our problem of describing and analyzing multi-spots. Throughout the paper, for (1.2) we assume that

$$
\begin{gathered}
\frac{\epsilon^{2}}{|\Omega|}<<1 \text { does not depend on } x, \\
\tau \geq 0 \text { does not depend on } x \text { or } \epsilon, \\
D, A>0 \text { do not depend on } x \text { (but may depend on } \epsilon \text { ), } \\
C_{1} \leq \frac{D}{|\Omega|}<<e^{C_{2} /(\epsilon / \sqrt{|\Omega|})} \text { for some } C_{1}>0,0<C_{2}<1 .
\end{gathered}
$$

To emphasize the dependence on $\epsilon$, we sometimes use the notations $D_{\epsilon}$ and $A_{\epsilon}$. We will make remarks on the relevance of these conditions towards the end of the introduction after explaining the main results.

What is a spot? It is a stationary localized structure for which the (slowly-diffusing) activator has a sharp peak and the other (fast-diffusing) component has a more shallow maximum (for activatorinhibitor systems) or minimum (for activator-substrate systems), respectively.

To describe a spot quantitatively, we first explain how the profile of the activator for a spot looks like in the Gierer-Meinhardt, Gray-Scott or Schnakenberg system. After re-scaling the spatial variable and the amplitude of the spot it is, to leading order, given by the solution $w$ of the following problem:

$$
\left\{\begin{array}{l}
\Delta w-w+w^{2}=0, \quad w>0 \text { in } R^{2}, \\
w(0)=\max _{y \in R^{2}} w(y), \quad w(y) \rightarrow 0 \text { as }|y| \rightarrow \infty .
\end{array}\right.
$$

The uniqueness of the solution $w$ was proved in [29]. By [16] we know that $w$ is rotationally symmetric. It is also important to note that

$$
w(y) \sim|y|^{-1 / 2} e^{-|y|} \quad \text { as }|y| \rightarrow \infty,
$$

so $w$ has exponential decay at infinity. In some sense, $w$ is the "ground state" to the multi-spot problem. If $f$ and $g$ have the more general form given in (1.1) one has to replace (1.3) by a more general problem. To simplify the presentation we only focus on this special case.

To get multi-spots from $w$, one re-scales space by the factor $\epsilon$, the amplitude by $\xi_{\epsilon}$ (depending on $\epsilon)$ and places spots at the positions $P_{j}^{\epsilon}$ which converge to some limiting positions $P_{j}^{0}$ as $\epsilon \rightarrow 0$. This is how we construct a good approximation to a solution. Then, using tools from nonlinear functional analysis, such as Liapunov-Schmidt reduction and fixed-point theorems, a multi-spot steady-state is established which is close to these multiple w's. This solves the existence problem.

Now we highlight the main ideas for solving the stability problem. This is done in two parts. 
Part I: Study of the eigenvalues with $\lambda_{\epsilon}=O(1)$,

Part II: Study of the eigenvalues with $\lambda_{\epsilon}=o(1)$ as $\epsilon \rightarrow 0$.

To establish Part I, we take the limit of the linearized operator to leading order as $\epsilon \rightarrow 0$. Then, using tools from nonlinear functional analysis such as nonlocal eigenvalue problems (NLEPs) and elliptic estimates we study the behavior of the eigenvalues of the limit problem for $\epsilon=0$. If $\lambda_{\epsilon}=O(1)$ by an argument of Dancer [5] we know that the stability behavior of the spectrum of the limit problem is the same as that for small $\epsilon$.

To establish Part II, the analysis used in Part I is not good enough for the eigenvalues with $\lambda_{\epsilon}=o(1)$ since knowing that their limit is zero does not tell us anything about the signs of the real parts of the eigenvalues for $\epsilon$ small. So a refined analysis is needed which goes beyond the leading order $O(1)$.

Since zero eigenvalues of the limit problem are connected with translation modes which belong to the kernel of the linearized operator, for small $\epsilon$ we have to deal with small eigenvalues and a corresponding approximate kernel. We use a projection similar to Liapunov-Schmidt reduction. This analysis leads to conditions on the positions of the multi-spots which are expressed in terms of the Green's function.

We now make a remark on the choice of diffusion constants. If $\epsilon / \sqrt{|\Omega|}$ is small enough the spots have radius of the order $\epsilon$ much smaller than the typical domain size (length-scale of the order $\sqrt{|\Omega|}$ ). So the spots are well separated and an explicit analysis is possible. The behavior for finite and not necessarily small $\epsilon / \sqrt{|\Omega|}$ is not so easy to capture analytically, and we have only been able to investigate it by numerical simulations (see Section 5).

The scaling given by the constants in the system can be reduced to the following two dimensionless parameters which are invariant under spatial scaling:

$$
\eta_{\epsilon}=\frac{1}{2 \pi} \frac{|\Omega|}{D_{\epsilon}} \log \frac{\sqrt{|\Omega|}}{\epsilon}, \quad \alpha_{\epsilon}=\frac{\epsilon^{2}}{|\Omega|} \frac{\int_{R^{2}} w^{2} d y}{A_{\epsilon}^{2}} .
$$

Note that $\eta_{\epsilon}$ describes the relative size of the diffusion constants scaled with respect to domain size and $\alpha_{\epsilon}$ measures the relative size of the activator diffusion constant scaled to domain size and the feed rate $A_{\epsilon}$, which is invariant under spatial scaling.

We prove that, in leading order as $\epsilon \rightarrow 0$ and for $\tau=0$, the maximal number $K$ of stable spots is given by

$$
K \leq\left(\frac{\eta_{\epsilon}}{\alpha_{\epsilon}}\right)^{1 / 2}=A_{\epsilon}\left(\frac{1}{2 \pi \int_{R^{2}} w^{2} d y} \frac{|\Omega|}{D_{\epsilon}} \frac{|\Omega|}{\epsilon^{2}} \log \frac{\sqrt{|\Omega|}}{\epsilon}\right)^{1 / 2} .
$$

Note that the r.h.s. in (1.6) may tend to infinity; in that case the number of spikes may become arbitrarily large if $\epsilon$ is chosen small enough and $D_{\epsilon}$ large enough. The borderline case of (1.6) giving a finite value is for

$$
\frac{D_{\epsilon}}{|\Omega|} \sim \log \frac{\sqrt{|\Omega|}}{\epsilon} \frac{|\Omega|}{\epsilon^{2}} A_{\epsilon}^{2} .
$$

The proof will show that in case there are more spots than described by (1.6) an overcrowding instability occurs which is linked to an eigenvalue of order $O(1)$ with positive real part. Dynamically, 
this implies that some of the spots will disappear on an $O(1)$ timescale due to overcrowding. We prove that (1.6) is true for $\tau=0$. Then, by a perturbation argument, it also holds if $\tau$ is small enough and independent of $\epsilon$ and $D_{\epsilon}$. For $\tau=0$ the eigenvalues become unstable by crossing the imaginary axis through zero.

Now we discuss the influence of the time-relaxation constant $\tau$ on the substrate. First we note that the one-spot solution is stable if $\tau$ is small. For increasing $\tau$ this spot may become unstable due to a Hopf bifurcation: The spot starts to oscillate, hereby disappearing and reappearing periodically. This means that if the substrate approximates a steady-state more slowly (increasing $\tau$ ) oscillations are more preferred. In the one-dimensional case this phenomenon has been studied analytically and numerically [72].

For multi-spots there are two competing instability mechanisms: Hopf bifurcation (which dominates for $\tau$ large) and overcrowding instability (which dominates for $K$ large).

Inequality (1.6), which is true for $\epsilon^{2} / \Omega$ sufficiently small, implies that the maximal number of stable spots increases if $A_{\epsilon}$ increases or if $D_{\epsilon} /|\Omega|$ decreases. These monotonicity properties are also observed numerically if $\epsilon^{2} / \Omega$ is finite and not necessarily very small.

We have simulated multi-spots numerically, and the results are presented in Section 5. Setting $\epsilon^{2}=0.001$ and varying $D$ we computed the Schnakenberg dynamics with feed rate $A=1$ and time relaxation constant $\tau=1$ on the unit disc with an initial condition having sixfold symmetry, see Figure 1. For $D / \epsilon^{2} \geq 10$, we have obtained multi-spots for the final state (i.e. the long-time limit) which is numerically stable. Further, the number of spots increases with decreasing $D$, see Figure 2 . If $D / \epsilon^{2}=5$ or smaller we did not observe multi-spots any more, but instead realized complex, chaotic dynamical behavior (not shown).

The problem with the quantitative application of our analytical results for finite $\epsilon / \sqrt{|\Omega|}$ is the following: They are derived taking into account $O(1)$ terms but neglecting terms of the order $O\left(\frac{1}{\log \frac{\sqrt{|\Omega|}}{\epsilon}}\right)$. However, if $\epsilon / \sqrt{|\Omega|}$ is not very small, then these two orders are comparable since $\left(\log \frac{|\Omega|}{\epsilon^{2}}\right)^{-1}$ decays only very slowly. We could improve the results by taking into account also contributions of the or$\operatorname{der} O\left(\frac{1}{\log \frac{|\Omega|}{\epsilon^{2}}}\right)$, but then the analysis becomes much more complicated since the positions and the amplitudes of the multi-spots are now both coupled with the diffusion constants.

Note that for the Gray-Scott system a constant similar to the one given in(1.6), which depends on $\eta_{\epsilon}$ and $\alpha_{\epsilon}$ only, determines the maximal number of stable spots.

For the Gierer-Meinhardt it is only $\eta_{\epsilon}$, that means the relative size of the two diffusion constants each scaled to domain size, which decides on the maximal number of stable spots. When the diffusion constant of the inhibitor becomes smaller, the maximal number of stable spots increases.

1.5. Structure of this paper. The structure of this paper is as follows: In Section 2 we state our main results. In Section 3 we prove the existence of multi-spots and determine the amplitudes and positions of the spots. In Section 4 we prove the stability of spots. In Section 5 we confirm our results 
by numerical simulations. In Section 6 we discuss our results. In three appendices we present the main technical tools: In Section 7/Appendix A we give an introduction to the Liapunov-Schmidt reduction which is used in Section 3. In Section 8/Appendix B we prove the stability of two nonlocal eigenvalue problems (NLEPs) which is needed in Subsection 4.1. In Section 9/Appendix C we study the small eigenvalues and these results are used in Subsection 4.2.

When technical details are omitted, they can be found in [75] or [76], even if we do not make explicit reference every time. To simplify our notation, we use e.s.t. to denote exponentially small terms (in their respective norms); more precisely, e.s.t. $=O\left(e^{-C_{2} /(\epsilon / \sqrt{|\Omega|})}\right)$ as $\epsilon \rightarrow 0$ for some $0<C_{2}<1$.

\section{Main Analytical Results: Existence and Stability of Multi-Spot Solutions}

We now present the main results of this paper about existence and stability of multi-spot solutions.

They will be explicitly given for the Schnakenberg system for which they are new. Then the earlier results for the Gierer-Meinhardt system and the Gray-Scott system will be summarized and all the results will be compared.

We assume that the diffusion constant of activator is small compared to the domain size, $0<$ $\epsilon^{2} /|\Omega|<<1$. This implies that the radius $\epsilon$ of spots will be small compared to the length scale $\sqrt{|\Omega|}$ of the domain. In this limit we are able to give an explicit analysis of existence and stability of multi-spots.

For the diffusion constant of the substrate in the limit $\epsilon \rightarrow 0$ we assume that $\lim _{\epsilon \rightarrow 0} \frac{D_{\epsilon}}{|\Omega|}=\frac{D_{0}}{|\Omega|} \in(0,+\infty]$ (note that this limit may be infinity). Let $\beta_{\epsilon}^{2}=\frac{|\Omega|}{D_{\epsilon}}$; then $\lim _{\epsilon \rightarrow 0} \beta_{\epsilon}=\beta_{0} \in[0,+\infty$ ).

We recall the two constants $\eta_{\epsilon}, \alpha_{\epsilon}$, describing the diffusion constant of the substrate and the feed rate, respectively, which were defined in (1.5):

$$
\eta_{\epsilon}=\frac{|\Omega|}{2 \pi D_{\epsilon}} \log \frac{\sqrt{|\Omega|}}{\epsilon}, \quad \alpha_{\epsilon}=\frac{\epsilon^{2}}{|\Omega|} \frac{\int_{R^{2}} w^{2} d y}{A_{\epsilon}^{2}} .
$$

Note that $\eta_{\epsilon}$ depends only on the two diffusion constants and is monotone decreasing in $D_{\epsilon} /|\Omega|$, so $\eta_{\epsilon}$ describes the growth rate of $D_{\epsilon} /|\Omega|$ as $\epsilon \rightarrow 0$. On the other hand, $\alpha_{\epsilon}$ depends on the diffusion constant of the activator and the feed rate. It is monotone decreasing in $A_{\epsilon}$, so $\alpha_{\epsilon}$ describes the growth rate of $A_{\epsilon}$ as $\epsilon \rightarrow 0$.

We consider the limits

$$
\eta_{0}=\lim _{\epsilon \rightarrow 0} \eta_{\epsilon} \in[0,+\infty], \quad \alpha_{0}=\lim _{\epsilon \rightarrow 0} \alpha_{\epsilon} \in[0,+\infty]
$$

and we assume that these limits exist (note that these limits may be zero or infinity).

For existence we assume that

$$
K^{2} \lim _{\epsilon \rightarrow 0} \frac{\alpha_{\epsilon}}{\eta_{\epsilon}} \neq 1
$$

where $K$ is the number of spots. We assume condition (T1) for the rest of the paper.

For stability we assume that

$$
K^{2} \lim _{\epsilon \rightarrow 0} \frac{\alpha_{\epsilon}}{\eta_{\epsilon}}<1
$$


where $K$ is the number of spots. Note that (T2) gives an explicit bound on the maximal number of stable spots. Further, $K$ increases if $\alpha_{\epsilon}$ decreases, i.e. if the feed rate $A_{\epsilon}$ increases, or if $\eta_{\epsilon}$ increases, i.e. if $D_{\epsilon} /|\Omega|$ decreases. In short, one has many stable spots if feeding dominates over diffusion, scaled with domain size, for the substrate.

Note that we sometimes write

in case this limit exists.

$$
\frac{\alpha_{0}}{\eta_{0}}=\lim _{\epsilon \rightarrow 0} \frac{\alpha_{\epsilon}}{\eta_{\epsilon}}
$$

With these notations, steady states for the Schnakenberg model

$$
\left\{\begin{array}{c}
v_{t}=\epsilon^{2} \Delta v_{\epsilon}-v_{\epsilon}+A_{\epsilon} u_{\epsilon} v_{\epsilon}^{2} \quad \text { in } \Omega \\
\tau u_{t}=D_{\epsilon} \Delta u_{\epsilon}+1-u_{\epsilon} v_{\epsilon}^{2} \quad \text { in } \Omega \\
\frac{\partial u_{\epsilon}}{\partial \nu}=\frac{\partial v_{\epsilon}}{\partial \nu}=0 \quad \text { on } \partial \Omega
\end{array}\right.
$$

are the solutions of the system

$$
\left\{\begin{array}{c}
\epsilon^{2} \Delta v_{\epsilon}-v_{\epsilon}+A_{\epsilon} u_{\epsilon} v_{\epsilon}^{2}=0 \quad \text { in } \Omega \\
D_{\epsilon} \Delta u_{\epsilon}+1-u_{\epsilon} v_{\epsilon}^{2}=0 \quad \text { in } \Omega \\
\frac{\partial u_{\epsilon}}{\partial \nu}=\frac{\partial v_{\epsilon}}{\partial \nu}=0 \quad \text { on } \partial \Omega
\end{array}\right.
$$

Note that we get (2.2) from (1.2) for the choice $\mu=0$ and after the re-scaling $\hat{v}=v, \hat{u}=A u$ (and then dropping hats). We make this re-scaling to simplify the calculation.

Now we describe the positions of the spots. Let $\mathbf{P}=\left(P_{1}, \ldots, P_{K}\right) \in \Omega^{K}$, where $\mathbf{P}$ is arranged such that

$$
\mathbf{P}=\left(P_{1}, P_{2}, \ldots, P_{K}\right) \text { with } P_{i}=\left(P_{i, 1}, P_{i, 2}\right) \quad \text { for } i=1,2, \ldots, K .
$$

For the rest of the paper we assume that the spots are well separated, which is made precise as follows:

$$
\begin{gathered}
\text { Assume that } \mathbf{P} \in \bar{\Lambda} \subset \Omega^{K} \text {, where for } \delta>0 \text { fixed we define } \\
\qquad=\left\{\left(P_{1}, P_{2}, \ldots, P_{K}\right) \in \Omega^{K}:\left|P_{i}-P_{j}\right|>2 \delta \text { for } i \neq j\right. \\
\text { and } \left.d\left(P_{i}, \partial \Omega\right)>\delta \text { for } i=1,2 \ldots, K\right\} .
\end{gathered}
$$

The positions of the spots can be determined explicitly. For this purpose, we introduce the Green's function $G(x, \xi)$ of the Laplace operator with Neumann boundary condition as follows:

$$
\begin{cases}\Delta G(x, \xi)-\frac{1}{|\Omega|}+\delta(x-\xi)=0, & x, \xi \in \Omega, \\ \int_{\Omega} G(x, \xi) d x=0, & \xi \in \Omega, \\ \frac{\partial G(x, \xi)}{\partial \nu_{x}}=0, & x \in \partial \Omega, \xi \in \Omega .\end{cases}
$$

Let

be the regular part of $G(x, \xi)$.

$$
H(x, \xi)=G(x, \xi)-\frac{1}{2 \pi} \log \frac{1}{|x-\xi|}
$$


For $\mathbf{P} \in \bar{\Lambda}$, we define

$$
F(\mathbf{P})=\sum_{k=1}^{K} H\left(P_{k}, P_{k}\right)+\sum_{i, j=1, \ldots, K, i \neq j} G\left(P_{i}, P_{j}\right)
$$

and

$$
M(\mathbf{P})=\nabla_{\mathbf{P}}^{2} F(\mathbf{P})
$$

Note that $F(\mathbf{P}) \in C^{\infty}(\bar{\Lambda})$.

For existence we assume that the positions of the spots approach a non-degenerate critical point of $F(\mathbf{P})$.

For stability we assume that the positions of the spots approach a non-degenerate local minimum point of $F(\mathbf{P})$.

After these preparations, we have all the notations in place which we need to formulate our results.

Our first main result concerns the existence of $K$-spot solutions.

Theorem 2.1. (Existence of $K$-spot solutions).

Suppose that $\lim _{\epsilon \rightarrow 0} \beta_{\epsilon}=\lim _{\epsilon \rightarrow 0}\left(\frac{|\Omega|}{D_{\epsilon}}\right)^{1 / 2} \geq 0$ and that (T1) holds. Assume that

(*) $\quad \mathbf{P}_{0}=\left(P_{1}^{0}, P_{2}^{0}, \ldots, P_{K}^{0}\right) \in \bar{\Lambda} \quad$ is a nondegenerate critical point of $F(\mathbf{P})$

(defined by (2.6)). Then, for $\epsilon$ sufficiently small, problem (2.3) has a multi-spot solution $\left(v_{\epsilon}, u_{\epsilon}\right)$ with the following properties:

(1) $v_{\epsilon}(x)=\sum_{j=1}^{K} \frac{1}{A_{\epsilon} \xi_{\epsilon}}\left(w\left(\frac{x-P_{j}^{\epsilon}}{\epsilon}\right)+O(h(\epsilon, \beta))\right)$ uniformly for $x \in \bar{\Omega}$,

$$
\xi_{\epsilon}=K \alpha_{\epsilon}(1+O(h(\epsilon, \beta))
$$

where

$$
h(\epsilon, \beta)=\max \left\{\frac{1}{\log \frac{\sqrt{|\Omega|}}{\epsilon}}, \beta^{2}\right\}
$$

(2) $u_{\epsilon}(x)=\xi_{\epsilon}(1+O(h(\epsilon, \beta))$ uniformly for $x \in \bar{\Omega}$.

(3) $P_{j}^{\epsilon} \rightarrow P_{j}^{0}$ as $\epsilon \rightarrow 0$ for $j=1, \ldots, K$.

Remarks: 1 . The case $\beta_{0}=0$ (i.e. $D_{\epsilon} /|\Omega| \rightarrow \infty$ ) is called the weak-coupling case. The case $\beta_{0}>0$ (i.e. $D_{\epsilon} /|\Omega| \rightarrow D_{0} /|\Omega|$ for some $\left.D_{0} /|\Omega| \in(0, \infty)\right)$ is called the strong-coupling case. The analysis is different in both cases and therefore these two cases have been considered separately. In this paper the main ideas of the proofs are explained for the weak-coupling case. We refer to [73] and [74] for a rigorous investigation of the strong-coupling case for the Gierer-Meinhardt system.

2. Note that the substrate has higher diffusion constant and so has a more shallow profile, which, to leading order, may be assumed to be constant in the weak-coupling case. In the strong-coupling case this assumption is not true anymore which makes the analysis more complicated.

Our second main result concerns the stability of the $K$-spot solutions constructed in Theorem 2.1. We say that an eigenvalue problem is (linearly) stable if there exists a constant $c_{0}>0$ such that for 
all eigenvalues $\lambda$, we have $\operatorname{Re}(\lambda) \leq-c_{0}$. We say it is (linearly) unstable if there exists an eigenvalue $\lambda$ with $\operatorname{Re}(\lambda)>0$.

Theorem 2.2. (Stability of $K$-spot solutions).

Let $\left(v_{\epsilon}, u_{\epsilon}\right)$ be the steady-state $K$-spot solutions of (2.2) constructed in Theorem 2.1 and let us assume that the conditions made in Theorem 2.1 are true. Further, we assume that

$$
(* *) \quad \mathbf{P}^{0} \text { is a nondegenerate local minimum point of } F(\mathbf{P}) .
$$

Then we have the following stability results:

Case 1. $\eta_{\epsilon} / \alpha_{\epsilon} \rightarrow 0$.

If $K=1$, there exists a unique $\tau_{1}>0$ such that for $\tau<\tau_{1},\left(u_{\epsilon}, v_{\epsilon}\right)$ is linearly stable, while for $\tau>\tau_{1}$, $\left(u_{\epsilon}, v_{\epsilon}\right)$ is linearly unstable.

If $K>1$, then $\left(u_{\epsilon}, v_{\epsilon}\right)$ is linearly unstable for any $\tau \geq 0$.

Case 2. $\eta_{\epsilon} / \alpha_{\epsilon} \rightarrow \infty$.

Then $\left(u_{\epsilon}, v_{\epsilon}\right)$ is linearly stable for any $\tau \geq 0$.

Case 3. $\eta_{\epsilon} / \alpha_{\epsilon} \rightarrow \eta_{0} / \alpha_{0} \in(0, \infty)$.

If condition (T2) holds, there exist $0<\tau_{2}<\tau_{3}$ such that $\left(u_{\epsilon}, v_{\epsilon}\right)$ is linearly stable for $0 \leq \tau<\tau_{2}$ or $\tau>\tau_{3}$.

If $K=1$ and $1>\frac{\eta_{0}}{\alpha_{0}}$, there exist $0<\tau_{4}<\tau_{5}$ such that $\left(u_{\epsilon}, v_{\epsilon}\right)$ is linearly stable for $\tau<\tau_{4}$ and linearly unstable for $\tau>\tau_{5}$.

If $K>1$ and $K^{2}>\frac{\eta_{0}}{\alpha_{0}}$, then $\left(u_{\epsilon}, v_{\epsilon}\right)$ is linearly unstable for all $\tau \geq 0$.

Remark: We are in the critical Case 3 of Theorem 2.2 if

$$
A_{\epsilon} \sim\left(\frac{D_{\epsilon}}{|\Omega|} \frac{\epsilon^{2}}{|\Omega|} \frac{1}{\log \frac{\sqrt{|\Omega|}}{\epsilon}}\right)^{1 / 2}
$$

(compare equation (1.6)).

Note that in the special case $\frac{D_{\epsilon}}{|\Omega|}=O(1)$ the critical rate for $A_{\epsilon}$ is $\left(\frac{\epsilon^{2}}{|\Omega|} \frac{1}{\log \frac{\sqrt{|\Omega|}}{\epsilon}}\right)^{1 / 2}$.

The chemical interpretation of this is the following: For the critical rate the feeding of substrate matches exactly the consumption.

2.1. Comparison of results with Gierer-Meinhardt system and Gray-Scott system. It is interesting to compare these results with previous results on the Gierer-Meinhardt and Gray-Scott systems.

For the Gierer-Meinhardt system the condition (T2) is replaced by

$$
K<\eta_{0}
$$

This says that with decreasing diffusion constant of the inhibitor the maximal number of stable spots increases. There is no feeding mechanism for activator-inhibitor systems and so only one constant plays a role. In short, one has many stable spots if the inhibitor diffuses slowly. 
For the Gray-Scott system the condition (T2) is replaced by

$$
\left(2 \eta_{0}+K\right)^{2}<\frac{\eta_{0}}{\alpha_{0}}
$$

Comparing this formula with (T2), there is an extra term $2 \eta_{0}$ on the left-hand side for Gray-Scott. So for the Gray-Scott system the maximal number of spots is smaller than for the Schnakenberg system. If $K>>\eta_{0}$ they are asymptotically the same.

Further, for Gray-Scott $K$ increases if $\alpha_{0}$ decreases, i.e. the feed rate increases. Interestingly, for Gray-Scott the dependence of $K$ from $\eta_{0}$ is not monotone: If $\eta_{0} \alpha_{0}<16$ then $K$ increases if $\eta_{0}$ increases, i.e. if $D_{0} /|\Omega|$ decreases. This is the same behavior as for Schnakenberg or Gierer-Meinhardt. However, if $\eta_{0} \alpha_{0}>16$ then $K$ decreases if $\eta_{0}$ increases, i.e. if $D_{0} /|\Omega|$ increases. This behavior is the reverse of Schnakenberg or Gierer-Meinhardt. So for Gray-Scott we have the most complicated behavior of the three as the diffusion and feeding mechanisms interact in an intricate way.

For all three systems condition (T1), or its equivalent, is obtained by making inequality (T2), or its equivalent, into an equality. Finally, for all three systems the function $F(\mathbf{P})$ (defined by (2.6)), which determines the positions of the spots, remains the same.

More comments on and explanations of these results can be found in the discussion section, Section 6.

\section{Existence Analysis}

In this section, we prove the existence result given in Theorem 2.1. We search for solutions of (2.3) in the following form

$$
v(x) \sim \sum_{j=1}^{K} \frac{1}{A \xi_{\epsilon, j}} w\left(\frac{x-P_{j}}{\epsilon}\right), \quad u\left(P_{j}\right)=\xi_{\epsilon, j},
$$

where $w$ is the unique solution of $(1.3),\left(P_{1}, \ldots, P_{K}\right) \in \Lambda, \xi_{\epsilon, j}$ is the amplitude of the $j-$ th spot and $P_{j}$ is the position of the $j-$ th spot.

In the first part, we determine, in the leading order, the equations for the amplitudes. In the second part, we use a Liapunov-Schmidt reduction procedure to find the positions for the spots.

3.1. Existence Proof I: Determining the amplitudes of the spots. In this section we compute the amplitudes of the spots, to leading order as $\epsilon \rightarrow 0$. It is found that, to leading order, the amplitudes depend on the number of spots but not on their positions. This computation depends very much on the reaction kinetics and for the Schnakenberg system is different from the other systems.

We first introduce some notation which we need to make a leading-order ansatz for a multi-spot solution of (2.3). Then we compute the unknown amplitudes of the spots.

We define cut-off functions as follows: Let $\chi$ be a smooth cut-off function which is equal to 1 in $B_{1}(0)$ and equal to 0 in $R^{2} \backslash \overline{B_{2}(0)}$, where $B_{r}(0)$ is the open ball with the origin as its center and radius $r$. 
With this notation in place, we can now make the following leading-order ansatz for a multi-spot solution $\left(v_{\epsilon}, u_{\epsilon}\right)$ of $(2.3)$ :

$$
\left\{\begin{array}{c}
v_{\epsilon}=\sum_{j=1}^{K} \frac{1}{A \xi_{\epsilon, j}} w\left(\frac{x-P_{j}}{\epsilon}\right) \chi_{\epsilon, j}(x)(1+h(\epsilon, \beta)), \\
u_{\epsilon}\left(P_{j}\right)=\xi_{\epsilon, j}(1+h(\epsilon, \beta)),
\end{array}\right.
$$

where $w$ is the unique solution of $(1.3),\left(P_{1}, \ldots, P_{K}\right) \in \Lambda, \xi_{\epsilon, j}$ is the amplitude of $u_{\epsilon}$ at $P_{j}$, and

$$
\chi_{\epsilon, j}(x)=\chi\left(\frac{x-P_{j}}{r_{0}}\right), \quad x \in \Omega, \quad j=1, \ldots, K .
$$

Note that the supports of $\chi_{\epsilon, j}$ and $\chi_{\epsilon, i}$ are disjoint for $i \neq j$.

From the second equation in (2.3), we get

$$
\Delta u_{\epsilon}+\frac{\beta^{2}}{|\Omega|}-\frac{\beta^{2}}{|\Omega|} u_{\epsilon} v_{\epsilon}^{2}=0 .
$$

Now, using (2.5) and the decomposition for the Green's function $G$ given after (2.5), we get from (3.4), for some unknown real constant $c$,

$$
\begin{gathered}
u_{\epsilon}\left(P_{i}\right)-c=\xi_{\epsilon, i}-c=\int_{\Omega} G\left(P_{i}, \xi\right) \frac{\beta^{2}}{|\Omega|}\left(1-u_{\epsilon}(\xi) v_{\epsilon}^{2}(\xi)\right) d \xi \\
=-\frac{1}{A^{2} \xi_{\epsilon, i}} \frac{\beta^{2}}{2 \pi} \frac{\epsilon^{2}}{|\Omega|} \log \frac{\sqrt{|\Omega|}}{\epsilon} \int_{R^{2}} w^{2}(y) d y(1+h(\epsilon, \beta)), \quad i=1, \ldots, K .
\end{gathered}
$$

Inserting the constants $\eta_{\epsilon}$ and $\alpha_{\epsilon}$ given in (1.5), from (3.5) we obtain the following system of equations for the amplitudes $\xi_{\epsilon, i}$ :

$$
\xi_{\epsilon, i}-c+\frac{\eta_{\epsilon} \alpha_{\epsilon}}{\xi_{\epsilon, i}}=O\left(\sum_{j=1}^{K} \frac{\beta^{2} \alpha_{\epsilon}}{\xi_{\epsilon, j}}\right), \quad i=1, \ldots, K
$$

for some unknown real constant $c$.

Integrating (3.4) over $\Omega$ and using the fact that $u_{\epsilon}$ satisfies the Neumann boundary condition, we get the solvability condition

This implies, using (3.2),

$$
|\Omega|=\int_{\Omega} u_{\epsilon}(\xi) v_{\epsilon}^{2}(\xi) d \xi
$$

$$
\sum_{j=1}^{K} \frac{1}{A^{2} \xi_{\epsilon, j}} \epsilon^{2} \int_{R^{2}} w^{2}(y) d y=|\Omega|(1+h(\epsilon, \beta)) .
$$

and (3.7) implies

$$
\sum_{j=1}^{K} \frac{\alpha_{\epsilon}}{\xi_{\epsilon, j}}=1+O(h(\epsilon, \beta)) .
$$

Our goal now is to solve the system (3.6), (3.8) for small $\epsilon$. We first consider the case $\epsilon=0$. Taking the limit $\epsilon \rightarrow 0$ in (3.6), (3.8), we get the following algebraic equations, where $\xi_{0}$ are the limits of the amplitudes $\xi_{\epsilon}$ as $\epsilon \rightarrow 0$ :

$$
\xi_{i}-c+\frac{\eta_{0} \alpha_{0}}{\xi_{i}}=0, \quad \sum_{i=1}^{K} \frac{\alpha_{0}}{\xi_{i}}=1
$$


for some unknown real constant $c$. Now we look for multi-spots of equal size. This means that $\xi_{i}$ should be independent of $i$. Denoting $\xi_{0}:=\xi_{i}, i=1,2, \ldots, K$, from the second equation of (3.9) we get

$$
\xi_{0}=K \alpha_{0}
$$

Then the first equation of (3.9) implies

$$
c=K \alpha_{0}+\frac{\eta_{0}}{K}
$$

The case of small $\epsilon$ can now be solved by the finite-dimensional version of the implicit function theorem under the assumption (T1), made throughout the paper.

This proves (2.8) in Theorem 2.1 and determines the amplitudes of the spots to leading order.

For other reaction kinetics, e.g. Gierer-Meinhardt of Gray-Scott, one has to solve similar, but different systems for the amplitudes.

3.2. Existence Proof II: Determining the positions of the spots. We complete the proof of Theorem 2.1 by rigorously determining the positions of the spots. Here an analysis is required which goes beyond the leading order. This is achieved in three steps.

Step 1. Choose good approximations to the solution. The main idea is to solve the second equation exactly for a good choice of the activator $v_{\epsilon}$ and then estimate the error in the first equation. An estimate, using the Green's function $G$ given in (2.5), provides the crucial step.

Step 2. If the spots are far apart they interact only weakly. Therefore they can be translated easily. These "translation modes" correspond to small eigenvalues in the linearized system. To eliminate these one has to project the corresponding $2 K$-dimensional kernel and co-kernel. This is achieved in a rigorous way using Liapunov-Schmidt reduction. This process effectively reduces the problem of finding multi-spots to a finite-dimensional problem of dimension $2 K$ for the positions of the spots.

Step 3. Solve the finite-dimensional problem derived in Step 2. One has to derive the finitedimensional problem explicitly and show that it can be solved using a finite-dimensional fixed-point theorem. Again the Green's function plays the central role. Checking the sufficiency conditions for the fixed-point theorem leads to the non-degeneracy condition $(*)$ in Theorem 2.1.

The details of the proof are given in Appendix A.

\section{Stability Analysis}

In this section, linearizing the system $(2.2)$ at the equilibrium states $\left(v_{\epsilon}, u_{\epsilon}\right)$ given in Theorem 2.1, we first derive the eigenvalue problem. There are two types of eigenvalues $\lambda_{\epsilon}=O(1)$ and $\lambda=o(1)$, respectively, and so the rest of the proof is divided into two parts.

Part I. $\lambda_{\epsilon}=O(1)$. Then, taking $\epsilon \rightarrow 0$, we derive the limit eigenvalue problem for $\epsilon=0$ which is a vectorial nonlocal eigenvalue problem (NLEP). By diagonalization we derive two scalar nonlocal eigenvalue problems (NLEPs) which will be analyzed in Appendix B.

If $\lambda_{0} \neq 0$, by an argument of Dancer [5], the stability problem for small $\epsilon>0$ is equivalent to stability in the limiting case $\epsilon=0$. 
Part II. $\lambda_{\epsilon}=o(1)$. In this case we have $\lambda_{0}=0$ and so the limiting eigenvalue does not give any information about the character of the eigenvalues for small $\epsilon>0$. Hence an analysis of the eigenvalue problem beyond the order $O(1)$ is required to understand the asymptotic behavior of the eigenvalues $\lambda_{\epsilon}=o(1)$ and determine their stability properties.

The argument is reminiscent of the Liapunov-Schmidt reduction considered in Subsection 3.2. The technical details of this analysis are presented in Appendix C.

We begin by deriving the eigenvalue problem. Here we use the same leading-order approximation of the solution as in Section 4, namely

$$
\left\{\begin{array}{c}
v_{\epsilon} \sim \sum_{i=1}^{K} \frac{1}{A \xi_{\epsilon, i}} w\left(\frac{x-P_{i}^{\epsilon}}{\epsilon}\right) \chi_{\epsilon, i}(x), \\
u_{\epsilon}\left(P_{i}^{\epsilon}\right) \sim \xi_{\epsilon, i}
\end{array}\right.
$$

where $\xi_{\epsilon, i} \sim \xi_{\epsilon} \sim K \alpha_{\epsilon}$ as computed in Section 4 .

Linearizing around the equilibrium states $\left(v_{\epsilon}, u_{\epsilon}\right)$

$$
\left\{\begin{array}{c}
v=v_{\epsilon}+\phi_{\epsilon}(y) e^{\lambda_{\epsilon} t} \\
u=u_{\epsilon}+\psi_{\epsilon}(x) e^{\lambda_{\epsilon} t}
\end{array}\right.
$$

and substituting the result into (2.2) we deduce the following eigenvalue problem

$$
\left\{\begin{array}{c}
\Delta_{y} \phi_{\epsilon}-\phi_{\epsilon}+2 A u_{\epsilon} v_{\epsilon} \phi_{\epsilon}+A \psi_{\epsilon} v_{\epsilon}^{2}=\lambda_{\epsilon} \phi_{\epsilon} \\
\frac{1}{\beta^{2}} \Delta \psi_{\epsilon}-2 u_{\epsilon} v_{\epsilon} \phi_{\epsilon}-\psi_{\epsilon} v_{\epsilon}^{2}=\tau \lambda_{\epsilon} \psi_{\epsilon}
\end{array}\right.
$$

Here $D=\frac{|\Omega|}{\beta^{2}}, \lambda_{\epsilon}$ is some complex number and

$$
\phi_{\epsilon} \in H_{N}^{2}\left(\Omega_{\epsilon}\right), \psi_{\epsilon} \in H_{N}^{2}(\Omega),
$$

where the index $N$ indicates that $\phi_{\epsilon}$ and $\psi_{\epsilon}$ satisfy no-flux boundary conditions and

$$
\Omega_{\epsilon}=\left\{y \in R^{2} \mid \epsilon y \in \Omega\right\} .
$$

4.1. Stability Part I: Large Eigenvalues. In the case $\lambda_{\epsilon}=O(1)$ we derive the vectorial NLEP and the two scalar NLEPs which will be investigated in Appendix B.

We compute the limit $\epsilon \rightarrow 0$ in the eigenvalue problem (4.2). The most important part here is to expand the second eigenfunction $\psi_{\epsilon}$, using the Green's function defined in (2.5).

Let

$$
\psi_{\epsilon}\left(P_{j}^{\epsilon}\right) \frac{1}{\xi_{j}^{2}}=\hat{\psi}_{\epsilon, j}, \quad \hat{\Psi}_{\epsilon}=\left(\hat{\psi}_{\epsilon, 1}, \ldots, \hat{\psi}_{\epsilon, K}\right) \rightarrow \hat{\Psi}_{0}
$$

Then we have

$$
\xi_{i}^{2} \hat{\psi}_{\epsilon, i}=\left[\frac{1}{\tau \lambda_{0}}\left(-2 A \alpha_{\epsilon} \frac{\sum_{j=1}^{K} \int_{R^{2}} w \phi_{\epsilon, j}}{\int_{R^{2}} w^{2}}-\alpha_{\epsilon} \sum_{j=1}^{K} \hat{\psi}_{\epsilon, j}\right)+\left(-2 A \eta_{\epsilon} \alpha_{\epsilon} \frac{\int_{R^{2}} w \phi_{i}}{\int_{R^{2}} w^{2}}-\eta_{\epsilon} \alpha_{\epsilon} \hat{\psi}_{\epsilon, i}\right)\right](1+o(1)) .
$$

Written in matrix notation, we obtain in the limit $\epsilon \rightarrow 0$

$$
\left[\left(\xi_{0}^{2}+\eta_{0} \alpha_{0}\right) \mathcal{I}+\frac{\alpha_{0}}{\tau \lambda_{0}} \mathcal{E}\right] \lim _{\epsilon \rightarrow 0} \hat{\Psi}_{0}=\left(-2 A \eta_{0} \alpha_{0} \mathcal{I}-\frac{2 A \alpha_{0}}{\tau \lambda_{0}} \mathcal{E}\right) \frac{\int_{R^{2}} w \Phi d y}{\int_{R^{2}} w^{2} d y},
$$


where $\mathcal{I}$ is the identity matrix,

$$
\mathcal{E}=\left(\begin{array}{ccc}
1 & \cdots & 1 \\
\vdots & \vdots & \vdots \\
1 & \cdots & 1
\end{array}\right)
$$

and

$$
\Phi=\left(\begin{array}{l}
\phi_{1} \\
\phi_{2} \\
\vdots \\
\phi_{K}
\end{array}\right) \in\left(H^{2}\left(R^{2}\right)\right)^{K}
$$

Thus for $\tau \lambda_{0} \neq 0$ in the limit $\epsilon \rightarrow 0$ from (4.2) we obtain the following vectorial nonlocal eigenvalue problem (NLEP):

$$
\Delta \Phi-\Phi+2 w \Phi-2 \mathcal{B} \frac{\int_{R^{2}} w \Phi}{\int_{R^{2}} w^{2}} w^{2}=\lambda_{0} \Phi
$$

where

$$
\mathcal{B}=\left[\left(\xi_{0}^{2}+\eta_{0} \alpha_{0}\right) \mathcal{I}+\frac{\alpha_{0}}{\tau \lambda_{0}} \mathcal{E}\right]^{-1}\left(\eta_{0} \alpha_{0} \mathcal{I}+\frac{\alpha_{0}}{\tau \lambda_{0}} \mathcal{E}\right)
$$

Note that we have expressed the matrix $\mathcal{B}$ explicitly. It is composed of the matrices $\mathcal{I}$ and $\mathcal{E}$. The constants depend on $\xi_{0}\left(=K \alpha_{0}\right), \eta_{0}, \alpha_{0}, \tau$.

More precisely, we have the following statement:

Theorem 4.1. Assume that $\left(v_{\epsilon}, u_{\epsilon}\right)$ satisfies (4.1).

Let $\lambda_{\epsilon}$ be an eigenvalue of (4.2) such that $\operatorname{Re}\left(\lambda_{\epsilon}\right)>-c_{0}$, where $0<c_{0}<1$.

(1) Suppose that (for suitable sequences $\epsilon_{n} \rightarrow 0$ ) we have $\lambda_{\epsilon_{n}} \rightarrow \lambda_{0} \neq 0$. Then $\lambda_{0}$ is an eigenvalue of the problem (NLEP) given in (4.6), (4.7).

(2) Let $\lambda_{0} \neq 0$ be an eigenvalue of the problem (NLEP) given in (4.6), (4.7). Then for $\epsilon$ sufficiently small, there is an eigenvalue $\lambda_{\epsilon}$ of (4.2) with $\lambda_{\epsilon} \rightarrow \lambda_{0}$ as $\epsilon \rightarrow 0$.

The proof of Theorem 4.1 is based on our derivation of the vectorial (NLEP) (4.6), (4.7) given before this theorem and the argument of Dancer [5].

Theorem 4.1 says that to analyze the character of eigenvalues $\lambda_{\epsilon}=O(1)$ for small $\epsilon$ it is enough to analyze them for $\lambda=0$. We will do this in the following.

Diagonalizing (4.6), (4.7), the study of large eigenvalues can be reduced to the study of the system of two scalar nonlocal eigenvalue problems (4.6), (4.7). To this end, we compute the eigenvalues of $\mathcal{B}$ :

$$
b_{1}=\frac{\eta_{0} \alpha_{0} \tau \lambda_{0}+K \alpha_{0}}{\left(\xi_{0}^{2}+\eta_{0} \alpha_{0}\right) \tau \lambda_{0}+K \alpha_{0}}, \quad b_{2}=\cdots=b_{K}=\frac{\eta_{0} \alpha_{0}}{\xi_{0}^{2}+\eta_{0} \alpha_{0}} .
$$

Note that the first eigenvalue is simple, the second eigenvalue has algebraic multiplicity $K-1$. 
Thus the study of the vectorial NLEP (4.6), (4.7) is reduced to the study of the following two scalar NLEPs:

$$
\Delta \Phi-\Phi+2 w \Phi-\frac{2\left(\eta_{0} \alpha_{0} \tau \lambda_{0}+K \alpha_{0}\right)}{\left(\xi_{0}^{2}+\eta_{0} \alpha_{0}\right) \tau \lambda_{0}+K \alpha_{0}} \frac{\int_{R^{2}} w \Phi}{\int_{R^{2}} w^{2}} w^{2}=\lambda_{0} \Phi
$$

and

$$
\Delta \Phi-\Phi+2 w \Phi-\frac{2 \eta_{0} \alpha_{0}}{\eta_{0} \alpha_{0}+\xi_{0}^{2}} \frac{\int_{R^{2}} w \Phi}{\int_{R^{2}} w^{2}} w^{2}=\lambda_{0} \Phi
$$

The two NLEPs (4.9) and (4.10) will be studied in Appendix B.

Remark: In the special case $\tau=0$ the proof of Theorem 4.1 has to be modified, using the Green's function $G$ defined in (2.5) and a solvability condition analogous to (3.7). Then one gets the result of Theorem 4.1 with (4.7) replaced by

$$
\mathcal{B}=\left[\left(\xi_{0}^{2}+\eta_{0} \alpha_{0}\right) \mathcal{I}-\frac{\xi_{0}^{2}}{K} \mathcal{E}\right]^{-1} \eta_{0} \alpha_{0} \mathcal{I}
$$

The eigenvalues of $\mathcal{B}$ in this case are

$$
b_{1}=1, \quad b_{2}=\ldots=b_{K}=\frac{\eta_{0} \alpha_{0}}{\xi_{0}^{2}+\eta_{0} \alpha_{0}} .
$$

These are the same eigenvalues as obtained by (formally) taking the limit $\tau \rightarrow 0$ in the eigenvalues for $\tau>0$. Note that for the Gierer-Meinhardt and Gray-Scott systems, respectively, this difficulty does not arise and there the cases $\tau>0$ and $\tau=0$ can be treated in a unified way.

4.2. Stability Part II: Small Eigenvalues. In this section we study the small eigenvalues, i.e., we assume that $\lambda_{\epsilon}=o(1)$. Small eigenvalues are important in the sense that they control the translational dynamics of the multi-spots.

This case uses a projection to approximate kernel and co-kernel which similar is similar to the Liapunov-Schmidt reduction presented in Appendix A. The details will be presented in Appendix C.

In particular, we will show in Appendix $\mathrm{C}$ that the eigenvalues $\lambda_{\epsilon}=o(1)$ are given by the eigenvalues of the following finite-dimensional problem:

$$
\begin{gathered}
\frac{\epsilon^{2}|\Omega| \alpha_{\epsilon} \beta_{\epsilon}^{2}}{2 \xi_{\epsilon}}\left(-\int_{R^{2}} w^{2} w^{\prime}|y| d y\right) \sum_{j=1}^{K} \sum_{k=1}^{2} a_{j, k}^{\epsilon}\left(\frac{\partial}{\partial P_{l, m}^{\epsilon}} \frac{\partial}{\partial P_{j, k}^{\epsilon}} F\left(\mathbf{P}^{\epsilon}\right)\right)+o\left(\epsilon^{2}|\Omega| \beta_{\epsilon}^{2} \sum_{j=1}^{K} \sum_{k=1}^{2}\left|a_{j, k}^{\epsilon}\right|\right) \\
=\lambda_{\epsilon} a_{l, m}^{\epsilon} \int_{R^{2}}\left(\frac{\partial w}{\partial y_{1}}\right)^{2} .
\end{gathered}
$$

Note the prominent role which is played in (4.12) by the function $F\left(\mathbf{P}^{\epsilon}\right)$ defined in (2.6). In fact, equation (4.12), up to scaling, is an eigenvalue problem for the matrix $M\left(\mathbf{P}^{\epsilon}\right)=\nabla_{\mathbf{P}^{\epsilon}}^{2} F\left(\mathbf{P}^{\epsilon}\right)$ defined in (2.7).

If condition $(* *)$ in Theorem 2.2 holds, then the symmetric matrix $M\left(\mathbf{P}_{0}\right)$ is strictly negative definite. Therefore $\operatorname{Re}\left(\lambda_{\epsilon}\right)<0$ for $\epsilon$ small. Thus we have proved that the small eigenvalues $\lambda_{\epsilon}=o(1)$ are stable if $\epsilon$ is small enough.

Combining the results for the large eigenvalues (Part I) and for the small eigenvalues (Part II), we have completed the proof of Theorem 2.2. 


\section{Confirmation of the Results by Numerical Simulations}

We present some numerical simulations using the Finite Element Software COMSOL Multiphysics. In particular, the simulations show that for smaller $D$ more stable spots are possible. For the simulations the original parabolic system (2.2) was implemented. The multi-spot plots shown are the activator concentration, reached as long-time limits and are numerically stable for the time-dependent problem.

In (2.2) we set $\epsilon^{2}=0.001, A=1$ and $\tau=1$. We take $\Omega$ to be the unit disk. For $D$ we choose the following values: $D=3.5,3.0,2.5,2.0,0.40,0.30,0.10,0.03,0.01$. Note that in the last case the ratio of the two diffusion coefficients is only 10. The number of stable spots in these cases are $K=3,4,5,6,6,11,18,19,24.5$ (counting boundary spots as half spots). Note the positions of the spots: In the first five cases they form regular polygons. In the sixth case we get a regular polygon plus two spots in the interior. In the seventh case we get two concentric polygons. In the eighth case we get two concentric polygons plus a spot in the middle. In the ninth case we get a more complicated configuration which shows some boundary spots as well as an almost hexagonal configuration of interior spots which have to be matched with the boundary spots. We display 2D projected plots as well as 3D plots.

The initial condition which has sixfold symmetry is shown in Figure 1. The final multi-spot states which are all numerically stable (shown for $t=10^{5}$ ) are displayed in Figure 2.

Of course, the initial condition does influence the dynamics. To emphasize other effects than the initial condition, we have kept it unchanged for all the simulations. The choice of initial condition will effect the time-dependent solution. It will also effect the final state, so that different initial conditions will a give different number of spots. After all, the results in Theorem 2.2 only give an upper bound on the number of spots, but all the solutions which have the maximal number or less, are stable if the positions of the spots are appropriate. Choosing other initial conditions, one would still get multi-spots as the final state, but slightly different numbers.

The simulations highlight the fact that for unchanged initial condition the number of spots in the long-time limit increases if the diffusion constant of the substrate decreases. Further, they show typical positions of the spots on a circular domain.

The mesh is not adaptive but mesh points have about equal distance throughout the domain. However, the mesh does not have any symmetry, e.g. radial symmetry. The mesh generally has a strong influence on the positions of spots (pinning effect of the numerical scheme) but only a minor impact on the number of spots or their shape. This effect can be seen in Figure 2, where the orientation of the 4-spot and 5-spot solutions is different from the initial condition given in Figure 1 and it is partly determined by the mesh. Note that for the 6-spot solutions in Figure 2 the orientation is the same as for the initial condition and in this case the mesh does not influence the positions of the spots. 
Initial condition (same for all $D$ )
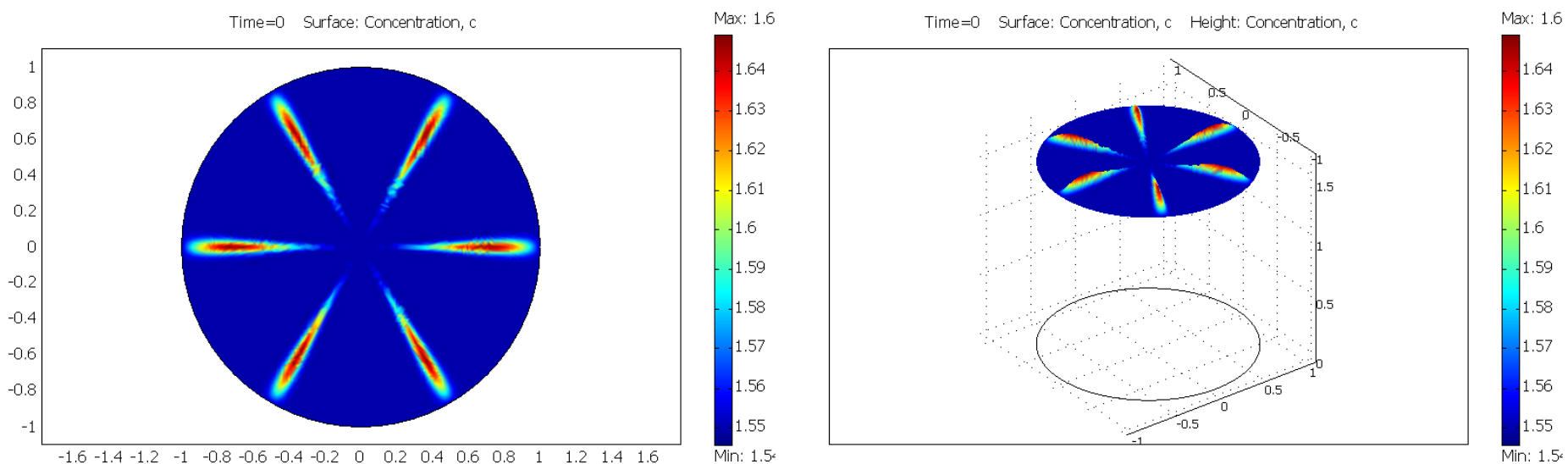

Figure 1. Initial condition (for $t=0$ ) on unit circle, for all $D$, given by

$$
a(x, y)=1.55+0.1 \sin \pi\left(x^{2}+y^{2}\right) \sin ^{100} \varphi,
$$

where $\varphi=\sin \left(\arcsin \left(\frac{x}{\sqrt{x^{2}+y^{2}}}\right)\right)$ is the angle with the $x$-axis. 
Final state for various $D$

$$
D=3.5
$$
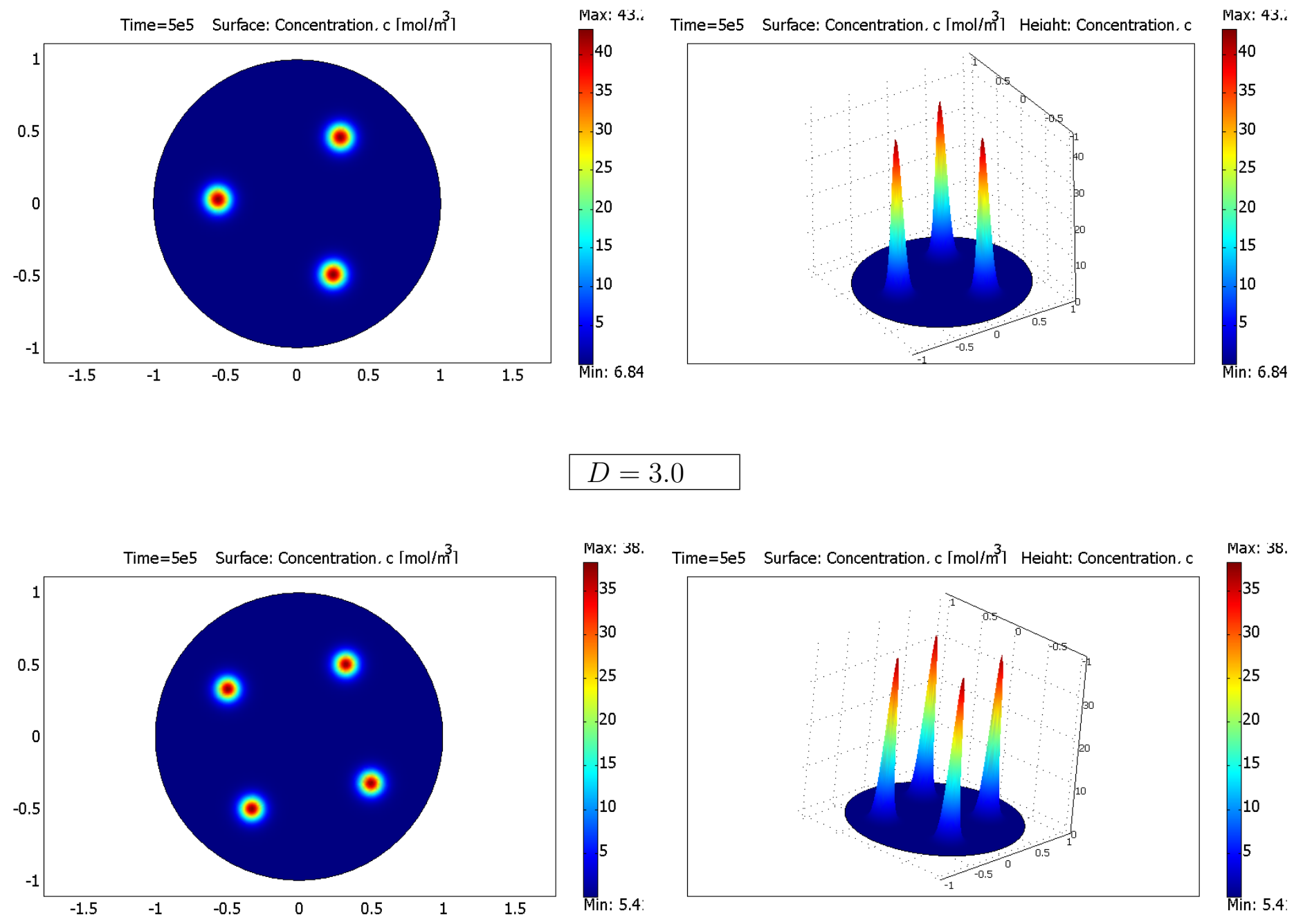

$$
D=2.5
$$
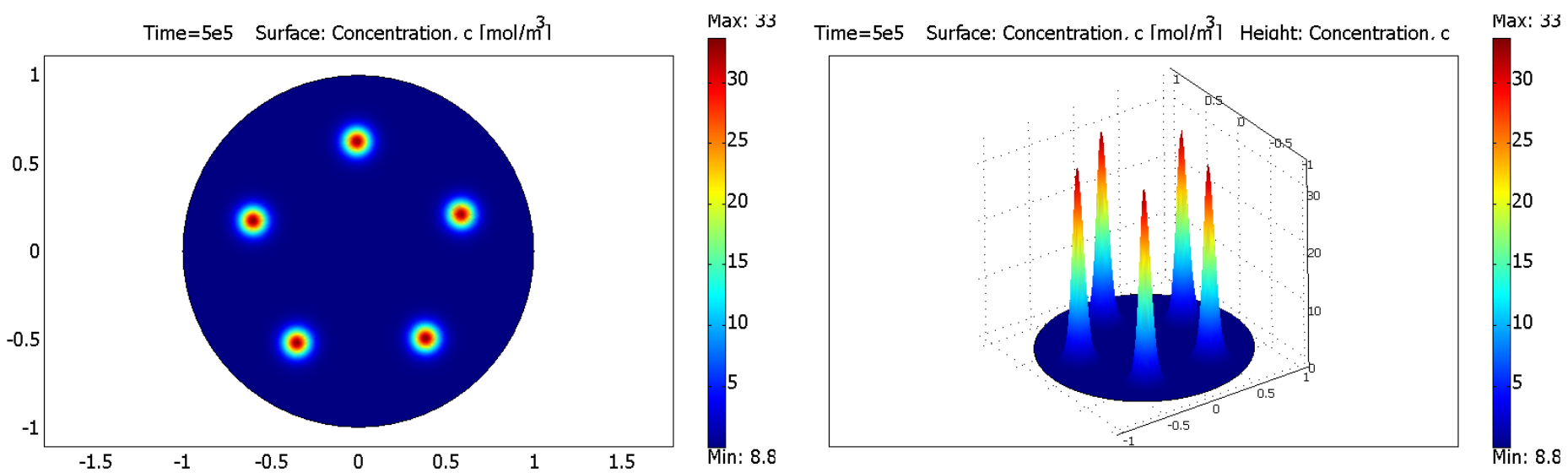

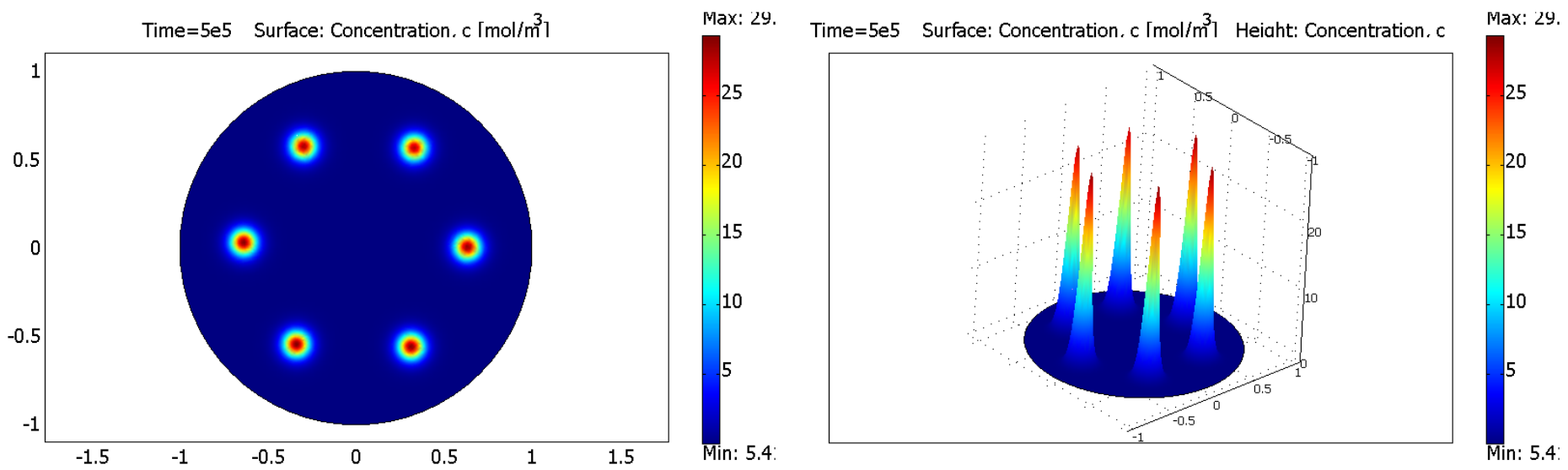

$$
D=0.40
$$
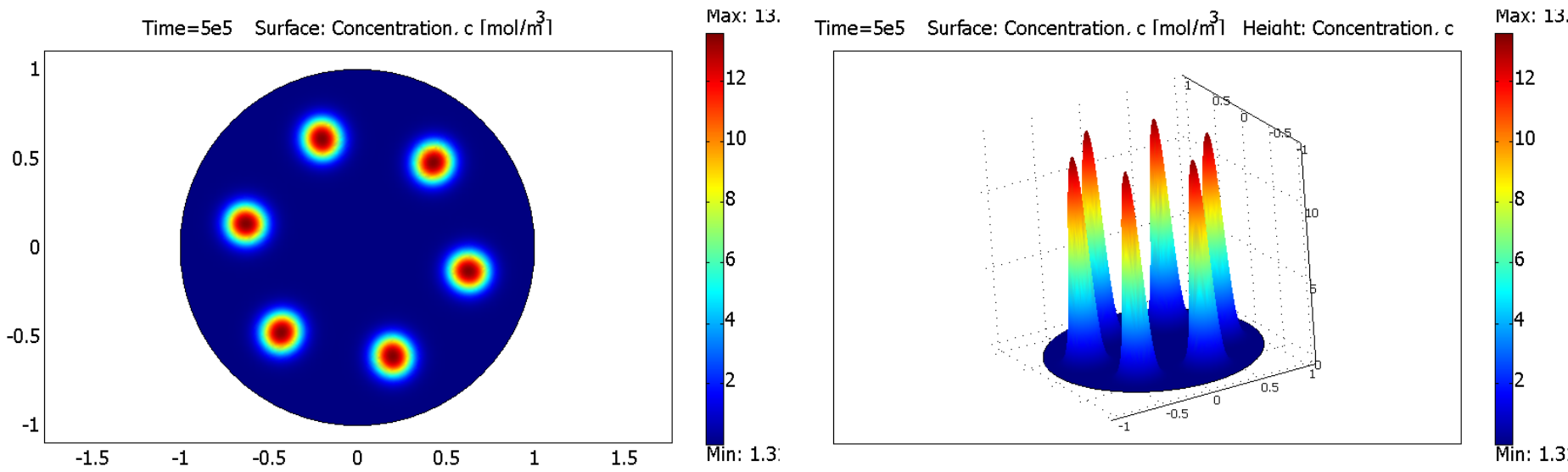

$$
D=0.30
$$
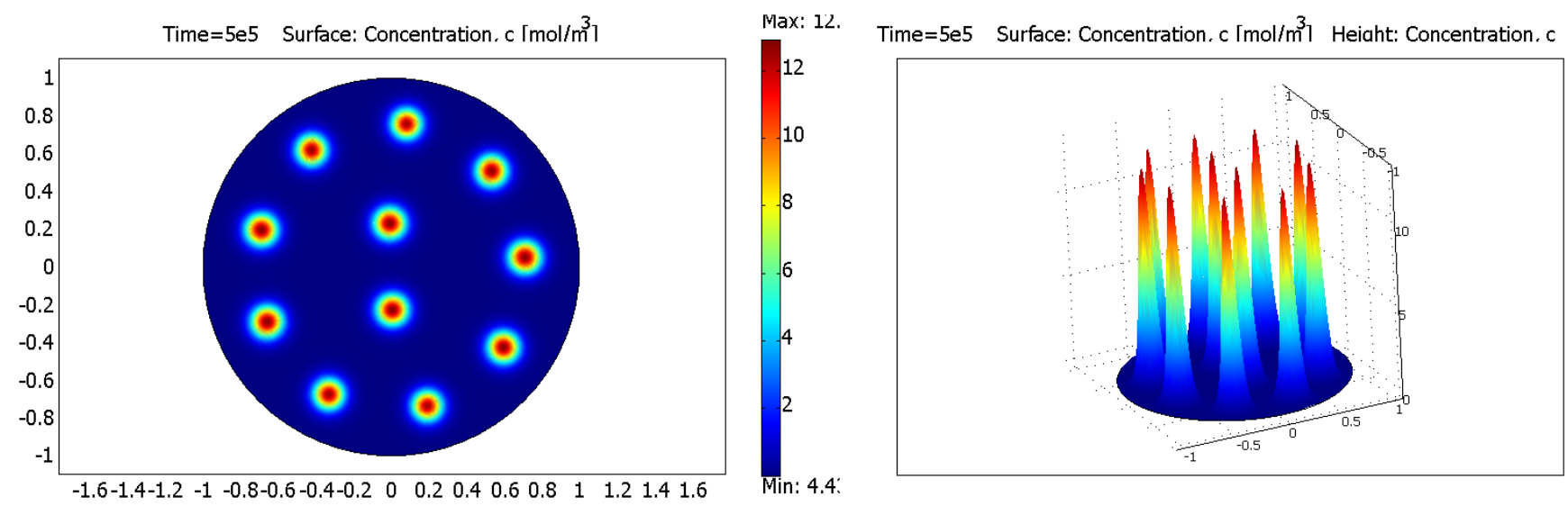

Max: 12.

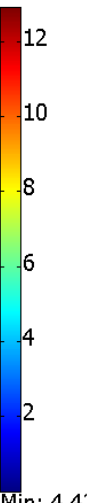

$D=0.10$ 
JUNCHENG WEI AND MATTHIAS WINTER
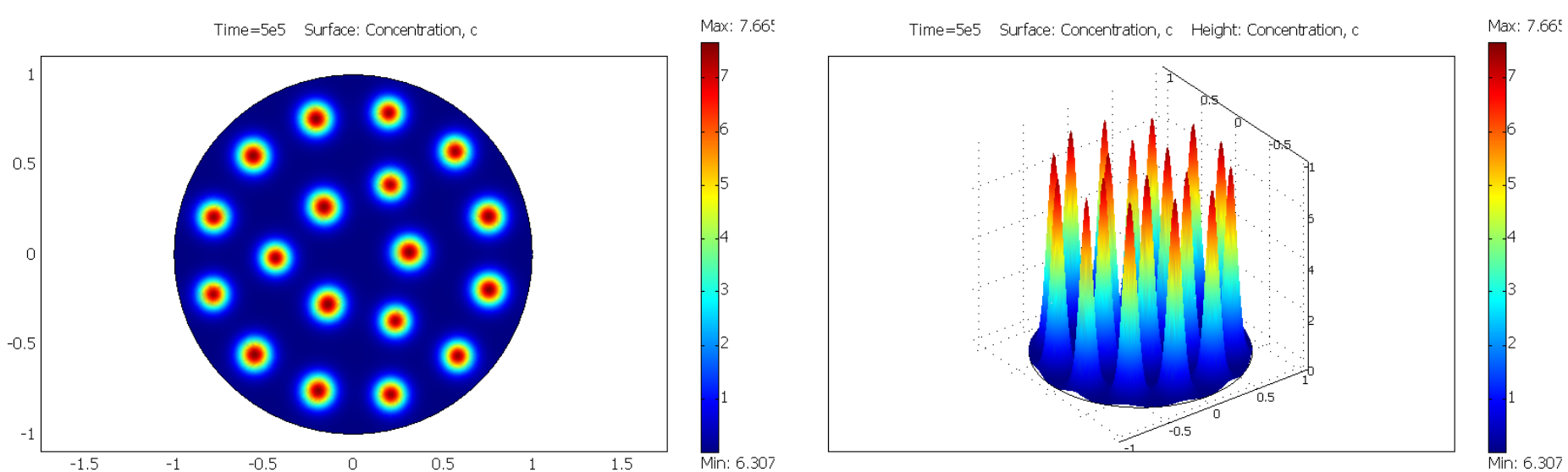


$$
D=0.03
$$
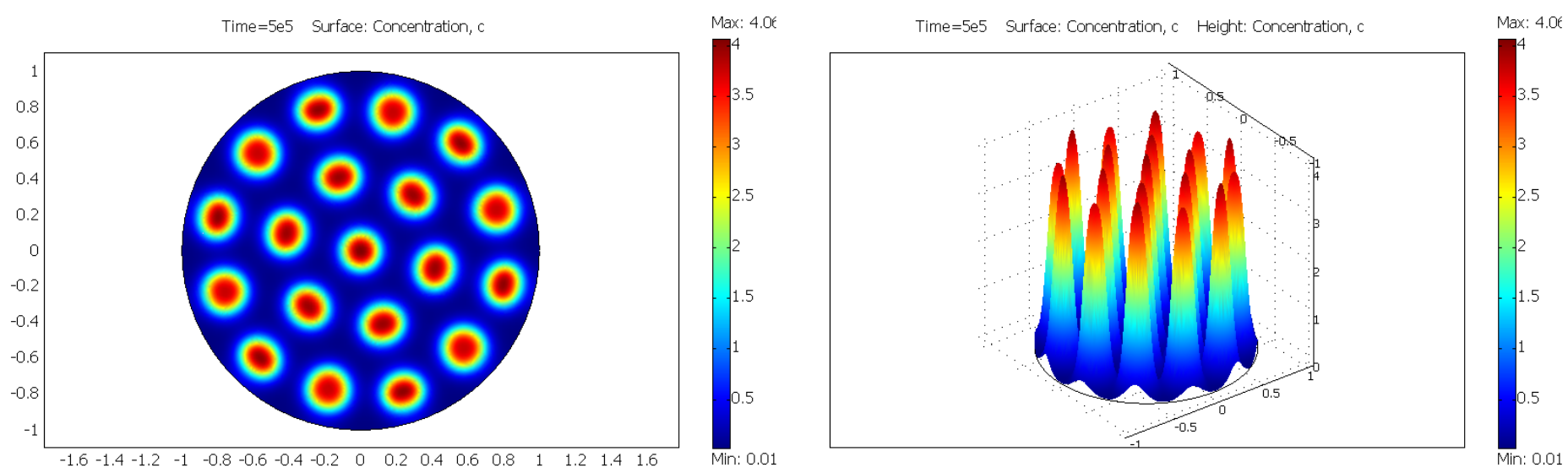

$$
D=0.01
$$
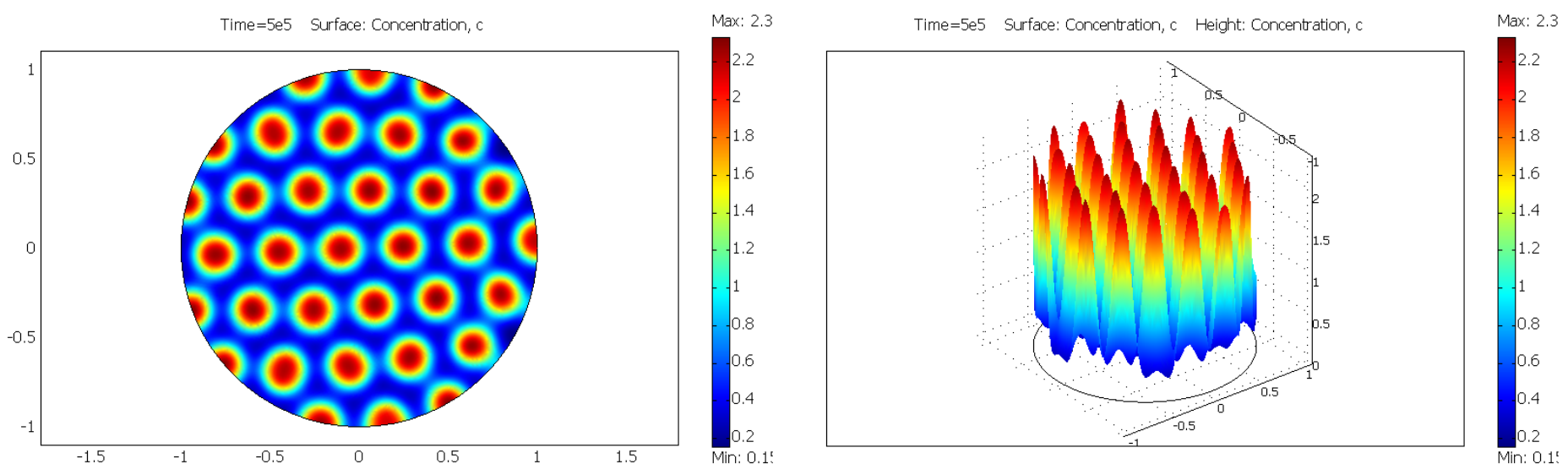

Figure 2. Plots for $D=3.5,3.0,2.5,2.0,0.40,0.30,0.10,0.03,0.01$. For decreasing $D$ more and more stable spots are possible. 


\section{Discussion}

We have studied multi-spot steady states for singularly perturbed activator-inhibitor systems (such as Gierer-Meinhardt) and activator-substrate systems (such as Gray-Scott and Schnakenberg), with particular emphasis on Schnakenberg, in a two-dimensional bounded domain.

A rigorous analysis for existence and stability of multi-spot steady states has been given. A result has been derived which states an upper bound on the maximal number of stable spots in terms of the diffusion constants and for activator-substrate systems also the feed rate (for vanishing or small enough time-relaxation constant of the inhibitor/substrate).

The results for Schnakenberg are new, whereas for Gierer-Meinhardt and Gray-Scott the results are reviewed.

All the results are rigorous if the diffusion constant of the activator is small enough, with respect to domain size. However, qualitatively, also for finite $\epsilon / \sqrt{|\Omega|}$, even if the maximal number of spots is not correctly predicted by our analytical results due to bad convergence properties of our method of proof, the monotonicity properties obtained (number of spots increases with decreasing diffusion constant of substrate and with increasing feed rate for Schnakenberg) are still correct.

A few remarks about the positions of spots are in order. The problem of finding the positions has been solved in our analysis by linking it to the Green's function. It can shown that the condition (**) given in Theorem 2.2 can be achieved generically. So for a given domain and given number of spots, if their number is not too large, one can generically find suitable positions for them such that the resulting configuration is stable. A more explicit relation to the geometry of the domain is desirable. For recent progress we refer to [23], [24].

An open problem is to get a better general understanding of the influence of the reaction-kinetics on the existence and stability of multi-spots. This is important since in biology or ecology many different types of reaction-kinetics, depending on the application, are being considered. We have studied three particular systems (Gierer-Meinhardt, Gray-Scott and Schnakenberg) which have two qualitatively different feed-back mechanisms (activator-inhibitor and activator-substrate). But this merely scratches the surface in gaining a full understanding of what will happen for general reaction kinetics. Can the reaction kinetics naturally be divided into classes which have similar behavior? What is the possible or typical behavior? Do large systems, which might be a better model of a biological system than a two-component or other small system, show many new effects or can they be well understood by reducing them to a few components which determine their behavior? Many questions remain and a lot of future work remains to be done to gain a better understanding of the full picture.

\section{Appendix A: The Liapunov-Schmidt Reduction}

The Liapunov-Schmidt reduction is a powerful tool of nonlinear functional analysis which has been used to prove the existence of multi-spot solutions for the Gierer-Meinhardt system [73], [74], [75] as well as for the Gray-Scott system [76], [77]. Here we will apply Liapunov-Schmidt reduction to the 
Schnakenberg system. We will focus on explaining the main ideas and refer to the above papers for complete proofs.

Step 1. Choose good approximations to the solution.

Let $\mathbf{P} \in \Lambda$ and $\left(\xi_{1}, \ldots, \xi_{K}\right)=\left(\xi_{0}, \ldots, \xi_{0}\right)$ where $\xi_{0}=K \alpha_{0}$. Now we choose good approximations to the solution. Let $\left(\xi_{\epsilon, 1}, \ldots, \xi_{\epsilon, K}\right)$ be such that $\left|\xi_{\epsilon, j}-\xi_{j}\right| \leq \delta_{0}$, where $\delta_{0}>0$ and $\delta_{0}$ is small enough. For the approximate solution use

$$
v_{\epsilon, \mathbf{P}}(y):=\sum_{j=1}^{K} \hat{v}_{\epsilon, j}(y)
$$

where

$$
v_{\epsilon, j}(y):=\frac{1}{A \xi_{\epsilon, j}} w\left(y-\frac{P_{j}}{\epsilon}\right) \chi_{\epsilon, j}(\epsilon y), \quad y \in \Omega_{\epsilon}:=\epsilon^{-1} \Omega
$$

and $\chi_{\epsilon, j}$ has been defined in (3.3). Note that $\xi_{\epsilon, j}$ is still undetermined. The second component, $u_{\epsilon, \mathbf{P}}$, is determined by solving the second component of the Schnakenberg system, which is linear in $u_{\epsilon, \mathbf{P}}$.

Using the Green's function derived in (2.5), the following result estimating the error in the first equation has been derived. (Recall the definition of $F(\mathbf{P})$ in $(2.6)$ ):

Lemma 7.1. For $x=P_{i}+\epsilon y,|\epsilon y|<\delta$, we have

$$
\Delta_{y} v_{\epsilon, \mathbf{P}}-v_{\epsilon, \mathbf{P}}+v_{\epsilon, \mathbf{P}}^{2} u_{\epsilon, \mathbf{P}}=: S_{1}\left(v_{\epsilon, \mathbf{P}}, u_{\epsilon, \mathbf{P}}\right)=S_{1,1}+S_{1,2},
$$

where

$$
S_{1,1}(y)=\beta^{2} \alpha_{\epsilon} \frac{1}{A \xi_{\epsilon, i}^{3}} w^{2}(y)\left(\epsilon \nabla_{P_{i}} F(\mathbf{P}) \cdot y+O\left(\epsilon \beta^{2}|y|+\epsilon^{2}|y|^{2}\right)\right)
$$

and

$$
S_{1,2}(y)=\frac{\beta^{2} \alpha_{\epsilon}}{A \xi_{\epsilon, i}^{3} \int_{R^{2}} w^{2}} w^{2}(y) \int_{R^{2}} \log \frac{|y-z|}{|z|} w^{2}(z) d z .
$$

Furthermore, $S_{1}\left(v_{\epsilon, \mathbf{P}}, u_{\epsilon, \mathbf{P}}\right)=$ e.s.t. for $\left|x-P_{j}\right| \geq \delta, j=1,2, \ldots, K$ and we have the estimate

$$
\left\|S_{1}\left(v_{\epsilon, \mathbf{P}}, u_{\epsilon, \mathbf{P}}\right)\right\|_{H^{2}\left(\Omega_{\epsilon}\right)}=O(h(\epsilon, \beta)) .
$$

Step 2. Use Liapunov-Schmidt reduction to derive a finite-dimensional problem for the positions of the spots.

We first study the linearized operator defined by

$$
\tilde{L}_{\epsilon, \mathbf{P}}: H_{N}^{2}\left(\Omega_{\epsilon}\right) \times H_{N}^{2}(\Omega) \rightarrow L^{2}\left(\Omega_{\epsilon}\right) \times L^{2}(\Omega), \quad \tilde{L}_{\epsilon, \mathbf{P}} \phi:=S_{\epsilon}^{\prime}\left(\begin{array}{c}
v_{\epsilon, \mathbf{P}} \\
u_{\epsilon, \mathbf{P}}
\end{array}\right) \phi
$$

where $\epsilon>0$ is small and $\mathbf{P} \in \bar{\Lambda}$.

Then the asymptotic limit of $\tilde{L}_{\epsilon, \mathbf{P}}$ as $\epsilon \rightarrow 0$ is given by the following system of linear operators

$$
\mathcal{L} \Phi:=\Delta \Phi-\Phi+2 w \Phi-2 \mathcal{B}_{0} \frac{\int_{R^{2}} w \Phi}{\int_{R^{2}} w^{2}} w^{2}, \quad \Phi=\left(\begin{array}{l}
\phi_{1} \\
\phi_{2} \\
\vdots \\
\phi_{K}
\end{array}\right) \in\left(H^{2}\left(R^{2}\right)\right)^{K}
$$


where

$$
\mathcal{B}_{0}=\left(\left(\xi_{0}^{2}+\alpha_{0} \eta_{0}\right) \mathcal{I}-\frac{\xi_{0}^{2}}{K} \mathcal{E}\right)^{-1} \alpha_{0} \eta_{0} \mathcal{I}
$$

and $\mathcal{E}$ is defined in (4.5). The eigenvalues of $\mathcal{B}_{0}$ are given by

$$
b_{1}=1, \quad b_{2}=\ldots=b_{K}=\frac{\eta_{0} \alpha_{0}}{\xi_{0}^{2}+\eta_{0} \alpha_{0}} .
$$

It is easy to see that $2 b_{1} \neq 1$ and $2 b_{2}=1$ if and only if $\eta_{0} \alpha_{0}=\xi_{0}^{2}$. This is excluded by assumption (T1).

Now we have the following lemma which states some key properties of the linear operator $\mathcal{L}$.

Lemma 7.2. Assume that assumption (T1) holds. Then

$$
\operatorname{Ker}(\mathcal{L})=\operatorname{Ker}\left(\mathcal{L}^{*}\right)=X_{0} \oplus X_{0} \oplus \cdots \oplus X_{0}
$$

where

$$
X_{0}=\operatorname{span}\left\{\frac{\partial w}{\partial y_{1}}, \frac{\partial w}{\partial y_{2}}\right\}
$$

and $\mathcal{L}^{*}$ is the adjoint operator of $\mathcal{L}$ under the $\left(L^{2}\left(R^{2}\right)\right)^{K}$ inner product.

As a consequence, the operator

$$
\mathcal{L}:\left(H^{2}\left(R^{2}\right)\right)^{K} \rightarrow\left(L^{2}\left(R^{2}\right)\right)^{K}
$$

is an invertible operator if it is restricted as follows

$$
\mathcal{L}:\left(X_{0} \oplus \cdots \oplus X_{0}\right)^{\perp} \cap\left(H^{2}\left(R^{2}\right)\right)^{K} \rightarrow\left(X_{0} \oplus \cdots \oplus X_{0}\right)^{\perp} \cap\left(L^{2}\left(R^{2}\right)\right)^{K} .
$$

Moreover, $\mathcal{L}^{-1}$ is bounded.

Proof: The result follows from the Fredholm Alternative Theorem.

Next we are going to reduce the infinite-dimensional problem to a finite-dimensional problem. Therefore, we now define an approximate kernel and an approximate co-kernel of the linear operator $\tilde{L}_{\epsilon, \mathbf{P}}$ by

$$
K_{\epsilon, \mathbf{P}}=C_{\epsilon, \mathbf{P}}=: \operatorname{span}\left\{\frac{\partial v_{\epsilon, \mathbf{P}}}{\partial P_{j, l}} \mid j=1, \ldots, K, l=1,2\right\}
$$

in $H_{N}^{2}\left(\Omega_{\epsilon}\right)$ and $L_{N}^{2}\left(\Omega_{\epsilon}\right)$, respectively, and we set

$$
\mathcal{K}_{\epsilon, \mathbf{P}}:=K_{\epsilon, \mathbf{P}} \oplus\{0\} \subset H_{N}^{2}\left(\Omega_{\epsilon}\right) \times H_{N}^{2}(\Omega), \mathcal{C}_{\epsilon, \mathbf{P}}:=C_{\epsilon, \mathbf{P}} \oplus\{0\} \subset L^{2}\left(\Omega_{\epsilon}\right) \times L^{2}(\Omega) .
$$

Let $\pi_{\epsilon, \mathbf{P}}$ denote the projection in $L^{2}\left(\Omega_{\epsilon}\right) \times L^{2}(\Omega)$ onto $\mathcal{C}_{\epsilon, \mathbf{P}}^{\perp}$, where the orthogonal complement is taken with the $L^{2}$ scalar product.

We are going to show that the equation

$$
\pi_{\epsilon, \mathbf{P}} \circ S_{\epsilon}\left(\begin{array}{c}
v_{\epsilon, \mathbf{P}}+\Phi_{\epsilon, \mathbf{P}} \\
u_{\epsilon, \mathbf{P}}+\Psi_{\epsilon, \mathbf{P}}
\end{array}\right)=0
$$

has the unique solution $\Sigma_{\epsilon, \mathbf{P}}=\left(\begin{array}{c}\Phi_{\epsilon, \mathbf{P}}(y) \\ \Psi_{\epsilon, \mathbf{P}}(x)\end{array}\right) \in \mathcal{K}_{\epsilon, \mathbf{P}}^{\perp}$ if $\epsilon$ is small enough, where the orthogonal complement is taken with the $L^{2}$ scalar product.

The following two propositions show the uniform invertibility of the corresponding linearized operator. 
Proposition 7.3. Let $\mathcal{L}_{\epsilon, \mathbf{P}}=\pi_{\epsilon, \mathbf{P}} \circ \tilde{L}_{\epsilon, \mathbf{P}}$. There exist positive constants $\bar{\epsilon}, \bar{\beta}, C$ such that for all $\epsilon \in$ $(0, \bar{\epsilon}), \beta \in(0, \bar{\beta})$,

$$
\left\|\mathcal{L}_{\epsilon, \mathbf{P}} \Sigma\right\|_{L^{2}\left(\Omega_{\epsilon}\right) \times L^{2}(\Omega)} \geq C\|\Sigma\|_{H^{2}\left(\Omega_{\epsilon}\right) \times H^{2}(\Omega)}
$$

for arbitrary $\mathbf{P} \in \bar{\Lambda}, \Sigma \in \mathcal{K}_{\epsilon, \mathbf{P}}^{\perp}$.

Proposition 7.4. There exist positive constants $\overline{\bar{\epsilon}}, \overline{\bar{\beta}}$ such that for all $\epsilon \in(0, \overline{\bar{\epsilon}}), \beta \in(0, \overline{\bar{\beta}})$ the map

$$
\mathcal{L}_{\epsilon, \mathbf{P}}=\pi_{\epsilon, \mathbf{P}} \circ \tilde{L}_{\epsilon, \mathbf{P}}: \mathcal{K}_{\epsilon, \mathbf{P}}^{\perp} \rightarrow \mathcal{C}_{\epsilon, \mathbf{P}}^{\perp}
$$

is surjective for arbitrary $\mathbf{P} \in \bar{\Lambda}$.

The main idea in the proofs of Propositions 7.3 and 7.4 is using an indirect argument and Lemma 7.2. For more details we refer to [75].

Using the Contraction Mapping Principle and recalling Lemma 7.1, we get

Lemma 7.5. There are $\bar{\epsilon}>0, \bar{\beta}, C>0$ such that for every triple $(\epsilon, \beta, \mathbf{P})$ with $0<\epsilon<\bar{\epsilon}, 0<\beta<\bar{\beta}$, $\mathbf{P} \in \bar{\Lambda}$ there exists a unique solution $\left(\Phi_{\epsilon, \mathbf{P}}, \Psi_{\epsilon, \mathbf{P}}\right) \in \mathcal{K}_{\epsilon, \mathbf{P}}^{\perp}$ satisfying $S_{\epsilon}\left(\begin{array}{c}v_{\epsilon, \mathbf{P}}+\Phi_{\epsilon, \mathbf{P}} \\ u_{\epsilon, \mathbf{P}}+\Psi_{\epsilon, \mathbf{P}}\end{array}\right) \in \mathcal{C}_{\epsilon, \mathbf{P}}$ Further, we have the estimate

$$
\left\|\left(\Phi_{\epsilon, \mathbf{P}}, \Psi_{\epsilon, \mathbf{P}}\right)\right\|_{H^{2}\left(\Omega_{\epsilon}\right) \times H^{2}(\Omega)} \leq C h(\epsilon, \beta) .
$$

Remark. The previous Lemma says that the function $\left(\Phi_{\epsilon, \mathbf{P}}, \Psi_{\epsilon, \mathbf{P}}\right) \in \mathcal{K}_{\epsilon, \mathbf{P}}^{\perp}$ solves the equation $S_{\epsilon}\left(\begin{array}{c}v_{\epsilon, \mathbf{P}}+\Phi_{\epsilon, \mathbf{P}} \\ u_{\epsilon, \mathbf{P}}+\Psi_{\epsilon, \mathbf{P}}\end{array}\right)=0$ up to a function which is contained in the finite-dimensional space $\mathcal{C}_{\epsilon, \mathbf{P}}$. We will solve this finite-dimensional problem in Step 3.

Step 3. Solve the finite-dimensional problem derived in Step 2.

By Lemma 7.5 there exists a unique solution $\left(\Phi_{\epsilon, \mathbf{P}}, \Psi_{\epsilon, \mathbf{P}}\right) \in \mathcal{K}_{\epsilon, \mathbf{P}}^{\perp}$ such that

$$
S_{\epsilon}\left(\begin{array}{c}
v_{\epsilon, \mathbf{P}}+\Phi_{\epsilon, \mathbf{P}} \\
u_{\epsilon, \mathbf{P}}+\Psi_{\epsilon, \mathbf{P}}
\end{array}\right) \in \mathcal{C}_{\epsilon, \mathbf{P}}
$$

Our idea is to find $\mathbf{P} \in \bar{\Lambda}$ such that

$$
S_{\epsilon}\left(\begin{array}{c}
v_{\epsilon, \mathbf{P}}+\Phi_{\epsilon, \mathbf{P}} \\
u_{\epsilon, \mathbf{P}}+\Psi_{\epsilon, \mathbf{P}}
\end{array}\right) \perp \mathcal{C}_{\epsilon, \mathbf{P}}
$$

Let

$$
W_{\epsilon}(\mathbf{P}):=\left(W_{\epsilon, 1,1}(\mathbf{P}), \ldots, W_{\epsilon, K, 2}(\mathbf{P})\right)
$$

where

$$
W_{\epsilon, j, i}(\mathbf{P}):=\frac{2 A \xi_{\epsilon, j}^{4}}{\alpha_{\epsilon} \beta_{\epsilon}^{2} \epsilon^{2}} \int_{\Omega_{\epsilon}} S_{1}\left(v_{\epsilon, \mathbf{P}}+\Phi_{\epsilon, \mathbf{P}}, u_{\epsilon, \mathbf{P}}+\Psi_{\epsilon, \mathbf{P}}\right) \frac{\partial v_{\epsilon, \mathbf{P}}}{\partial P_{j, i}} d y .
$$

We calculate, using the expansion of the Green's function,

$$
W_{\epsilon}(\mathbf{P})=c_{0} \nabla_{\mathbf{P}} F(\mathbf{P})(1+O(h(\epsilon, \beta))),
$$


where

$$
c_{0}=-\int_{R^{2}} w^{2} \frac{\partial w}{\partial y_{i}} y_{i} d y=\frac{1}{3} \int_{R^{2}} w^{3} d y
$$

Now we conclude the proof by applying some tools from nonlinear functional analysis.

Suppose that for $\mathbf{P}_{0}$ we have $\nabla_{\mathbf{P}} F\left(\mathbf{P}_{0}\right)=0$, $\operatorname{det}\left(\nabla_{\mathbf{P}}^{2}\left(F\left(\mathbf{P}_{0}\right)\right) \neq 0\right.$, then, since $W_{\epsilon}$ is continuous and for $\epsilon, \beta$ sufficiently small maps balls into (possibly larger) balls, the standard Brouwer's fixed point theorem shows that for $\epsilon<<1$ there exists a $\mathbf{P}^{\epsilon}$ such that $W_{\epsilon}\left(\mathbf{P}^{\epsilon}\right)=0$ and $\mathbf{P}^{\epsilon} \rightarrow \mathbf{P}_{0}$.

Thus we have proved the following proposition.

Proposition 7.6. For $\epsilon$ sufficiently small there exist points $\mathbf{P}^{\epsilon}$ with $\mathbf{P}^{\epsilon} \rightarrow \mathbf{P}_{\mathbf{0}}$ such that $W_{\epsilon}\left(\mathbf{P}^{\epsilon}\right)=0$.

Finally, we complete the proof of Theorem 2.1.

Proof of Theorem 2.1: By Proposition 7.6, there exists $\mathbf{P}^{\epsilon} \rightarrow \mathbf{P}_{0}$ such that $W_{\epsilon}\left(\mathbf{P}^{\epsilon}\right)=0$. Let $v_{\epsilon}=v_{\epsilon, \mathbf{P}^{\epsilon}}+\Phi_{\epsilon, \mathbf{P}^{\epsilon}}, u_{\epsilon}=u_{\epsilon, \mathbf{P}^{\epsilon}}+\Psi_{\epsilon, \mathbf{P}^{\epsilon}}$. It is easy to see that $u_{\epsilon}=\xi_{\epsilon, j}(1+O(h(\epsilon, \beta)))$ and hence $v_{\epsilon} \geq 0$. By the Maximum Principle, $v_{\epsilon}>0$. Therefore $\left(v_{\epsilon}, u_{\epsilon}\right)$ satisfies Theorem 2.1.

This concludes the rigorous proof of the existence of multi-spot steady states.

\section{Appendix B: Study of Two Nonlocal Eigenvalue Problems}

In this appendix, we give a rigorous study of the nonlocal eigenvalue problems (4.9) and (4.10). To this end, we write them in a unified form:

$$
L \phi:=\Delta \phi-\phi+2 w \phi-f\left(\tau \lambda_{0}\right) \frac{\int_{R^{2}} w \phi}{\int_{R^{2}} w^{2}} w^{2}=\lambda_{0} \phi, \quad \phi \in H^{2}\left(R^{2}\right),
$$

where $w$ is the unique solution of $(1.3)$,

$$
f\left(\tau \lambda_{0}\right)=\frac{2\left(\eta_{0} \alpha_{0} \tau \lambda_{0}+K \alpha_{0}\right)}{\left(\xi_{0}^{2}+\eta_{0} \alpha_{0}\right) \tau \lambda_{0}+K \alpha_{0}} \quad \text { and } f\left(\tau \lambda_{0}\right)=\frac{2 \eta_{0} \alpha_{0}}{\xi_{0}^{2}+\eta_{0} \alpha_{0}}
$$

for (4.9) and (4.10), respectively. Note that $f$ is a continuous function.

We will study these NLEPs in a sequence of lemmas, where the main results are Lemma 8.4 and Lemma 8.5.

We begin with the following auxiliary lemma about instability for small $f(0)$.

Lemma 8.1. If $f(0)<1$ and $0<c \leq f(\alpha)$ for $\alpha>0$, then there exists a positive eigenvalue of (8.1) for any $\tau \geq 0$.

Proof: This result was introduced and proved in [76]. The main ideas of the proof are as follows: First the algebraic equation

$$
\int_{R^{2}} w^{2}=f\left(\tau \lambda_{0}\right) \int_{R^{2}}\left[\left(\left(L_{0}-\lambda_{0}\right)^{-1} w^{2}\right) w\right] d y
$$

is derived and it is shown that it is equivalent to (8.1). Then, using certain identities for $w$ and the intermediate value theorem, it is shown that there is a positive solution of (8.2) and hence a positive eigenvalue of (8.1). 
Similarly, we have the following result about instability if $f$ is small for large arguments.

Lemma 8.2. If $\lim _{\tau \rightarrow+\infty} f(\tau \lambda)=f_{+\infty}<1$ and $0<c \leq f(\alpha)$ for $\alpha>0$, there exists a positive eigenvalue of (8.1) for $\tau>0$ large.

Proof: Similar to Lemma 8.1.

The following lemma summarizes the result if $f$ is a real constant.

Lemma 8.3. Consider the eigenvalue problem

$$
\Delta \phi-\phi+2 w \phi-\gamma \frac{\int_{R^{2}} w \phi}{\int_{R^{2}} w^{2}} w^{2}=\lambda_{0} \phi, \quad \phi \in H^{2}\left(R^{2}\right),
$$

where $w$ is the unique solution of (1.3) and $\gamma$ is real.

(1) If $\gamma>1$, there exists a positive constant $c_{0}$ such that $\operatorname{Re}\left(\lambda_{0}\right) \leq-c_{0}$ for any nonzero eigenvalue $\lambda_{0}$ of $(8.3)$.

(2) If $\gamma<1$, there exists a positive eigenvalue $\lambda_{0}$ of (8.3).

(3) If $\gamma \neq 1$ and $\lambda_{0}=0$, then $\phi \in \operatorname{span}\left\{\frac{\partial w}{\partial y_{1}}, \frac{\partial w}{\partial y_{2}}\right\}$.

(4) If $\gamma=1$ and $\lambda_{0}=0$, then $\phi \in \operatorname{span}\left\{w, \frac{\partial w}{\partial y_{1}}, \frac{\partial w}{\partial y_{2}}\right\}$.

Proof: (1), (3) and (4) have been proved in Theorem 5.1 of [69]. (2) follows from Lemma 8.1.

Now we consider the function $f(\tau \lambda)=\frac{\mu}{1+\tau \lambda}$. We then have

Lemma 8.4. Let $\gamma=\frac{\mu}{1+\tau \lambda_{0}}$ where $\mu>0, \tau \geq 0$.

(1) Suppose that $\mu>1$. Then there exists a unique $\tau=\tau_{1}>0$ such that for $\tau>\tau_{1}$, (8.1) admits a positive eigenvalue, and for $\tau<\tau_{1}$, all eigenvalues of problem (8.1) satisfy $\operatorname{Re}(\lambda)<0$. At $\tau=\tau_{1}, L$ has a Hopf bifurcation.

(2) Suppose that $\mu<1$. Then $L$ admits a real eigenvalue $\lambda_{0}$ with $\lambda_{0} \geq c_{2}>0$.

\section{Proof of Lemma 8.4:}

(2) follows from Lemma 8.1 and (1) was proved in [76]. The main ideas of the proof are to write the eigenvalue problem as a system of two real algebraic equations at the Hopf bifurcation point $\lambda_{0}=\lambda_{I} \sqrt{-1}$, then applying the intermediate value theorem to one of the these equations to compute the eigenvalue and finally using the other equation to compute (the unique) $\tau$.

Now we consider (8.1) with $f(\tau \lambda)=\frac{2\left(\eta_{0} \alpha_{0} \tau \lambda+K \alpha_{0}\right)}{\left(\eta_{0} \alpha_{0}+\xi_{0}^{2}\right) \tau \lambda+K \alpha_{0}}$ and $0<\tau<\infty$. We have

Lemma 8.5. Let

$$
\begin{gathered}
a=\frac{6 \eta_{0}^{2} \alpha_{0}^{2}\left(\eta_{0} \alpha_{0}-\xi_{0}^{2}\right)^{2} \int_{R^{2}} w^{4}}{\left(\xi_{0}^{2}+\eta_{0} \alpha_{0}\right)^{2} \int_{R^{2}} w^{2}}, \quad b=\frac{8 K \eta_{0}^{2} \alpha_{0}^{3} \xi_{0}^{2} \int_{R^{2}} w^{4}}{\left(\xi_{0}^{2}+\eta_{0} \alpha_{0}\right)^{2} \int_{R^{2}} w^{2}}, \\
c=\frac{3}{2} K^{2} \alpha_{0}^{2}
\end{gathered}
$$


and let $0<\tau_{2} \leq \tau_{3}$ be the two solutions (if they exist) of the following quadratic equation

$$
a \tau^{2}-b \tau+c=0
$$

(1) If $\eta_{0} \alpha_{0}>\xi_{0}^{2}$, then for $\tau<\tau_{2}$ or $\tau>\tau_{3}$ problem (8.1) with $f(\tau \lambda)=\frac{2\left(\eta_{0} \alpha_{0} \tau \lambda+K \alpha_{0}\right)}{\left(\eta_{0} \alpha_{0}+\xi_{0}^{2}\right) \tau \lambda+K \alpha_{0}}$ is stable. (If there are no solutions of (8.5) then this problem is stable for all $\tau \geq 0$.)

(2) If $\eta_{0} \alpha_{0}<\xi_{0}^{2}$, for $\tau$ small problem (8.1) with $f(\tau \lambda)=\frac{2\left(\eta_{0} \alpha_{0} \tau \lambda+K \alpha_{0}\right)}{\left(\eta_{0} \alpha_{0}+\xi_{0}^{2}\right) \tau \lambda+K \alpha_{0}}$ is stable while for $\tau$ large it is unstable.

Remarks: 1. Equation (8.5) may not have a solution if $\eta_{0} \alpha_{0}$ is large. It is also easy to see that

$$
0<\tau_{2} \leq \tau_{3}:=\frac{b}{a}=\frac{4 \xi_{0}^{2} K \alpha_{0}}{3\left(\eta_{0} \alpha_{0}-\xi_{0}^{2}\right)^{2}} .
$$

2. While $\tau_{2}$ and $\tau_{3}$ are rigorous estimates of the true values they are in general not optimal. from above and from below

Proof: We prove (1) first.

We first multiply the nonlocal eigenvalue problem by $\bar{\phi}$ and integrate to express it as a quadratic form. This quadratic form is simplified in two steps: first multiplying the nonlocal eigenvalue problem by $w$, integrating and using the resulting identity; second considering the real part of the eigenvalue problem and using an inequality for quadratic forms (see Lemma B.1 in [69]).

After these two steps we get the following inequality:

$$
\frac{3}{2} K^{2} \alpha_{0}^{2}+\left(\frac{3}{2}\left(\eta_{0} \alpha_{0}-\xi_{0}^{2}\right)^{2} \tau^{2}-2 \tau K \alpha_{0} \xi_{0}^{2}\right) \lambda_{I}^{2} \leq 0
$$

$\lambda_{R} \geq 0$, where $\lambda=\lambda_{R}+\sqrt{-1} \lambda_{I}$ and we have assumed that under the assumption that $\lambda_{R} \geq 0$.

If $\tau \geq \tau_{4}$, then (8.7) does not hold. To study the case $\tau<\tau_{4}$, we need to have an upper bound for $\lambda_{I}$. The quadratic form of the eigenvalue problem gives the estimate

$$
\lambda_{I} \int_{R^{2}}|\phi|^{2} d y=\operatorname{Im}\left(-f(\tau \lambda) \frac{\int_{R^{2}} w \phi d y}{\int_{R^{2}} w^{2} d y} \int_{R^{2}} w^{2} \bar{\phi}\right) d y
$$

Hence, Schwartz inequality gives

$$
\left|\lambda_{I}\right| \leq|f(\tau \lambda)| \sqrt{\frac{\int_{R^{2}} w^{4} d y}{\int_{R^{2}} w^{2} d y}} \leq \frac{2 \eta_{0} \alpha_{0}}{\xi_{0}^{2}+\eta_{0} \alpha_{0}} \sqrt{\frac{\int_{R^{2}} w^{4} d y}{\int_{R^{2}} w^{2} d y}} .
$$

Substituting (8.8) into (8.7), $\lambda_{I}$ is eliminated, and we see that if

$$
a \tau^{2}-b \tau+c>0
$$

where $a, b, c$ are defined in (8.4), then (8.7) is impossible.

We next prove (2). For $\tau$ large, it follows that $f(\tau \lambda) \rightarrow f_{+\infty}:=\frac{2 \eta_{0} \alpha_{0}}{\xi_{0}^{2}+\eta_{0} \alpha_{0}}<1$, then the perturbation argument of Dancer [5] shows that there exists a real and positive eigenvalue of (8.1) with $f(\tau \lambda)=$ $\frac{2\left(\eta_{0} \alpha_{0} \tau \lambda+K \alpha_{0}\right)}{\left(\eta_{0} \alpha_{0}+\xi_{0}^{2}\right) \tau \lambda+K \alpha_{0}}$. For $\tau$ small, the proof follows by the argument in (1). 


\section{Appendix C: The small eigenvalues}

We shall analyze the small eigenvalues $\lambda_{\epsilon}=o(1)$ and determine if they give rise to an instability or not.

Let us first define

$$
\tilde{v}_{\epsilon, j}\left(y-\frac{P_{j}^{\epsilon}}{\epsilon}\right)=\chi_{\epsilon, j}(x) \hat{v}_{\epsilon}(y), \quad j=1, \ldots, K,
$$

where $\chi_{\epsilon, j}$ was defined in $(3.3)$ and $\hat{v}_{\epsilon} \in H_{N}^{2}\left(\Omega_{\epsilon}\right)$.

Similar to the Liapunov-Schmidt reduction in Appendix A we decompose the eigenfunction of the activator, $\phi_{\epsilon}$, as follows:

$$
\phi_{\epsilon}=\sum_{j=1}^{K} \sum_{k=1}^{2} a_{j, k}^{\epsilon} \frac{\partial \tilde{v}_{\epsilon, j}}{\partial y_{k}}+\phi_{\epsilon}^{\perp}
$$

with real numbers $a_{j, k}^{\epsilon}$, where

$$
\phi_{\epsilon}^{\perp} \perp K_{\epsilon, \mathbf{P}^{\epsilon}}^{n e w}=\operatorname{span}\left\{\frac{\partial \tilde{v}_{\epsilon, j}}{\partial y_{k}} \mid j=1, \ldots, K, k=1,2\right\} \subset H_{N}^{2}\left(\Omega_{\epsilon}\right) .
$$

Accordingly, we put

$$
\psi_{\epsilon}(x)=\sum_{j=1}^{K} \sum_{k=1}^{2} a_{j, k}^{\epsilon} \psi_{\epsilon, j, k}+\psi_{\epsilon}^{\perp}
$$

by solving the second equation of the system which is linear in $\psi_{\epsilon, j, k}$.

Suppose that $\left\|\phi_{\epsilon, j}\right\|_{H^{2}\left(\Omega_{\epsilon}\right)}=1$. Then $\left|a_{j, k}^{\epsilon}\right| \leq C$. Now the main idea is to first show that $\phi_{\epsilon}^{\perp}$ is small (Step 1) and then to obtain algebraic equations for $a_{j, k}^{\epsilon}$ (Step 2).

We begin with

Step 1: Estimates for $\phi_{\epsilon}^{\perp}$.

Substituting the decompositions (9.9) of $\phi_{\epsilon}$ and (9.10) of $\psi_{\epsilon}$ into (4.2), we have

$$
\begin{gathered}
\sum_{j=1}^{K} \sum_{k=1}^{2} a_{j, k}^{\epsilon}\left(\tilde{v}_{\epsilon, j}\right)^{2}\left[\psi_{\epsilon, j, k}-\epsilon \frac{\partial u_{\epsilon}}{\partial x_{k}}\right]+\Delta_{y} \phi_{\epsilon}^{\perp}-\phi_{\epsilon}^{\perp}+2 \hat{v}_{\epsilon} u_{\epsilon} \phi_{\epsilon}^{\perp}+\left(\hat{v}_{\epsilon}\right)^{2} \psi_{\epsilon}^{\perp}-\lambda_{\epsilon} \phi_{\epsilon}^{\perp} \\
=\lambda_{\epsilon} \sum_{j=1}^{K} \sum_{k=1}^{2} a_{j, k}^{\epsilon} \frac{\partial \tilde{v}_{\epsilon, j}}{\partial y_{k}} .
\end{gathered}
$$

Since $\phi_{\epsilon}^{\perp} \perp K_{\epsilon, \mathbf{P}^{\epsilon}}^{n e w}$, then by an argument similar to the proof of Lemma 7.5 it follows that to estimate $\left\|\phi_{\epsilon}^{\perp}\right\|_{H^{2}\left(\Omega_{\epsilon}\right)}$ it is enough to $\psi_{\epsilon, l, k}-\epsilon \frac{\partial u_{\epsilon}}{\partial x_{k}}$ near $x \in B_{r_{0}}\left(P_{l}^{\epsilon}\right)$. This is possible by expanding the Green's function. We refer to [75] and [76] for details.

Step 2: Algebraic equations for $a_{j, k}^{\epsilon}$.

Multiplying both sides of (9.11) by $-\frac{\partial \tilde{v}_{\epsilon, l}}{\partial y_{m}}$ and integrating over $\Omega_{\epsilon, P_{l}^{\epsilon}}$, we obtain

$$
\begin{gathered}
\text { r.h.s. }=\lambda_{\epsilon} \sum_{j=1}^{K} \sum_{k=1}^{2} a_{j, k}^{\epsilon} \int_{\Omega_{\epsilon, P_{l}^{\epsilon}}} \frac{\partial \tilde{v}_{\epsilon, j}}{\partial y_{k}} \frac{\partial \tilde{v}_{\epsilon, l}}{\partial y_{m}} d y=\frac{1}{\xi_{\epsilon, l}^{2}} \lambda_{\epsilon} \sum_{j, k} a_{j, k}^{\epsilon} \delta_{j l} \delta_{k m} \int_{R^{2}}\left(\frac{\partial w}{\partial y_{1}}\right)^{2} d y(1+o(1)) \\
=\frac{1}{\xi_{\epsilon, l}^{2}} \lambda_{\epsilon} a_{l, m}^{\epsilon} \int_{R^{2}}\left(\frac{\partial w}{\partial y_{1}}\right)^{2} d y(1+o(1))
\end{gathered}
$$


and

$$
\begin{gathered}
\text { l.h.s. }=\epsilon^{2} \sum_{j=1}^{K} \sum_{k=1}^{2} a_{j, k}^{\epsilon} \int_{\Omega_{\epsilon, P_{l}^{\epsilon}}}\left(\tilde{v}_{\epsilon, j}\right)^{2}\left[\psi_{\epsilon, j, k}-\epsilon \frac{\partial u_{\epsilon}}{\partial x_{k}}\right] \frac{\partial \tilde{v}_{\epsilon, l}}{\partial y_{m}} d y+o\left(\beta^{2} \epsilon^{2}|\Omega| \sum_{j=1}^{K} \sum_{k=1}^{2}\left|a_{j, k}^{\epsilon}\right|\right) \\
=\frac{\epsilon^{2}|\Omega| \beta_{\epsilon}^{2} \alpha_{\epsilon}}{\xi_{\epsilon, j}^{3}} \int_{R^{2}} w^{2} \frac{\partial w}{\partial y_{m}} y_{m} d y \sum_{j=1}^{K} \sum_{k=1}^{2} a_{j, k}^{\epsilon}\left(-\frac{\partial}{\partial P_{l, m}^{\epsilon}} \frac{\partial}{\partial P_{j, k}^{\epsilon}} F\left(\mathbf{P}^{\epsilon}\right)\right) \\
+o\left(\beta_{\epsilon}^{2} \epsilon^{2}|\Omega| \sum_{j=1}^{K} \sum_{k=1}^{2}\left|a_{j, k}^{\epsilon}\right|\right),
\end{gathered}
$$

using the Green's function to expand $\psi_{\epsilon, j, k}$.

Applying some identities for $w$ and combining l.h.s. and r.h.s., the finite-dimensional eigenvalue problem (4.12) follows.

Acknowledgments. The research of JW is supported by an Earmarked Grant from RGC of Hong Kong. The research of MW is supported by a BRIEF Award of Brunel University. MW thanks the Department of Mathematics at CUHK for their kind hospitality.

\section{REFERENCES}

[1] D. L. Benson, P. K. Maini, J. A. Sherratt, Unravelling the Turing bifurcation using spatially varying diffusion coefficients, J. Math. Biol. 37 (1998), 381-417.

[2] V. Castets, E. Dulos, J. Boissonade and P. De Kepper, Experimental evidence of a sustained standing Turing-type nonequilibrium chemical pattern, Phys. Rev. Lett. 64 (1990), 2953-2956.

[3] E. J. Crampin, E. A. Gaffney and P. K. Maini, Reaction and diffusion on growing domains: Scenarios for robust pattern formation, Bull. Math. Biol. 61 (1999), 1093-1120.

[4] E. J. Crampin, E. A. Gaffney and P. K. Maini, Mode doubling and tripling in reaction-diffusion patterns on growing domains: a piece-wise linear model, J. Math. Biol. 44 (1999), 107-128. 1093-1120.

[5] E.N. Dancer, On stability and Hopf bifurcations for chemotaxis systems, Methods Appl. Anal. 8 (2001), 245-256.

[6] P. De Kepper, V. Castets, E. Dulos and J. Boissonade, Turing-type chemical pattern in the chlorite-iodide-malonic acid reaction, Physica D 49 (1991), 161-169.

[7] A. Doelman, R.A. Gardner, and T.J. Kaper, Large stable pulse solutions in reaction-diffusion equations, Indiana Univ. Math. J. 50 (2001), 443-507.

[8] A. Doelman, A. Gardner and T.J. Kaper, Stability analysis of singular patterns in the 1-D Gray-Scott model: A matched asymptotic approach, Phys. D 122 (1998), 1-36.

[9] A. Doelman, A. Gardner and T.J. Kaper, A stability index analysis of 1-D patterns of the Gray-Scott model, Mem. Amer. Math. Soc. 155 (2002), no. 737, xii+64 pp.

[10] A. Doelman, T. Kaper, and P. A. Zegeling, Pattern formation in the one-dimensional Gray-Scott model, Nonlinearity 10 (1997), 523-563.

[11] V. Dufiet and J. Boissonade, Conventional and unconventional Turing patterns, J. Chem. Phys. 96 (1992), 664-673.

[12] S. Ei, The motion of weakly interacting pulses in reaction-diffusion systems, J. Dynam. Diff. Equations 14 (2002), 85-87.

[13] S. Ei, Y. Nishiura and K. Ueda, $2^{n}$ splitting or edge splitting: a manner of splitting in dissipative systems, Jap. J. Ind. Appl. Math. 18 (2001), 181-205.

[14] P. C. Fife, Stationary patterns for reaction-diffusion systems, In Nonlinear Diffusion, Research Notes in Math., Vol. 14, pp. 81-121, Pitman, London (1977).

[15] P. C. Fife, Large time behaviour of solutions of bistable nonlinear diffusion equations, Arch. Rational Mech. Anal. 70 (1979), 31-46.

[16] B. Gidas, W.M. Ni, and L. Nirenberg, Symmetry of positive solutions of nonlinear elliptic equations in $R^{N}, A d v$. Math. Suppl. Stud. 7A (1981), 369-402.

[17] A. Gierer and H. Meinhardt, A theory of biological pattern formation, Kybernetik (Berlin) 12 (1972), 30-39.

[18] P. Gray and S. K. Scott, Autocatalytic reactions in the isothermal, continuous stirred tank reactor: isolas and other forms of multistability, Chem. Eng. Sci. 38 (1983), 29-43. 
[19] P. Gray and S. K. Scott, Autocatalytic reactions in the isothermal, continuous stirred tank reactor: oscillations and instabilites to the system $A+2 B \rightarrow 3 B, B \rightarrow C$, Chem. Eng. Sci. 39 (1984), 1087-1097.

[20] J.K. Hale, L.A. Peletier and W.C. Troy, Exact homoclinic and heteroclinic solutions of the Gray-Scott model for autocatalysis, SIAM J. Appl. Math. 61 (2000), 102-130.

[21] J.K. Hale, L.A. Peletier and W.C. Troy, Stability and instability of the Gray-Scott model: the case of equal diffusion constants, Appl. Math. Letters 12 (1999), 59-65.

[22] D. Iron, J. Wei and M. Winter, Stability analysis of Turing patterns generated by the Schnakenberg model, J. Math. Biol. 49 (2004), 358-390.

[23] T. Kolokolnikov and M.J. Ward, Reduced wave Green's functions and their effect on the dynamics of a spike for the Gierer-Meinhardt model. Eur. J. Appl. Math. 14 (2003), 513-545.

[24] T. Kolokolnikov and M.J. Ward, Bifurcation of spike equilibria in the near-shadow Gierer-Meinhardt model. Discrete Contin. Dyn. Syst. Ser. B 4 (2004), 1033-1064.

[25] T. Kolokolnikov, M.J. Ward and J. Wei, The existence and stability of spike equilibria in the one-dimensional Gray-Scott model: the low-feed regime. Stud. Appl. Math. 115 (2005), 21-71.

[26] T. Kolokolnikov, M.J. Ward and J. Wei, The existence and stability of spike equilibria in the one-dimensional Gray-Scott model: the pulse-splitting regime. Physica D 202 (2005), 258-293.

[27] S. Kondo and R. Asai, A viable reaction-diffusion wave on the skin of Pomacanthus, a marine Angelfish. Nature 376 (1995), 765-768.

[28] A.J. Koch and H. Meinhardt, biological pattern formation from basic mechanisms to complex structures, Rev. Modern Physics 66(2994), 1481-1507.

[29] M.K. Kwong and L. Zhang, Uniqueness of positive solutions of $\Delta u+f(u)=0$ in an annulus, Differential Integral Equations 4 (1991), 583-599.

[30] I. Lengyel and I. R. Epstein, Modeling of Turing structures in the Chlorite-Iodide- Malonic Acid-Starch Reaction System, Science 251 (1991), 650-652.

[31] K. J. Lee, W. D. McCormick, J. E. Pearson, and H. L. Swinney, Experimental observation of self-replicating spots in a reaction-diffusion system, Nature 369 (1994), 215-218.

[32] K. J. Lee, W. D. McCormick, Q. Ouyang, and H. L. Swinney, Pattern formation by interacting chemical fronts, Science 261 (1993), 192-194.

[33] S. A. Levin, The problem of pattern and scale in ecology, Ecology 73 (1992), 1943-1967.

[34] A. Madzvamuse, P. K. Maini and A. J. Wathen, A moving grid finite element method for the simulation of pattern generation by Turing models on growing domains, J. Sci. Comput. 24 (2005), 247-262.

[35] A. Madzvamuse, A.J. Wathen and P. K. Maini, A moving grid finite element method applied to a model biological pattern generator, J. Comput. Phys. 190 (2003), 478-500.

[36] P.K. Maini, R.E. Baker and C.M. Chuong, The Turing model comes of molecular age, Science 314 (2006), $1397-1398$.

[37] P.K. Maini, K.J. Painter and H. Chau, Spatial pattern formation in chemical and biological systems, J. Chem. Soc., Faraday Trans. 93 (1997), 3601-3610.

[38] H. Meinhardt, Model of Biological Pattern Formation, Academic Press, London, 1982.

[39] H. Meinhardt, The Algorithmic Beauty of Sea Shells, Springer-Verlag, Berlin, 1995.

[40] M. Mimura, Reaction-diffusion systems arising in biological and chemical systems: applications of singular limit procedures. In: Mathematical Aspects of Evolving Interfaces (Funchal, 2000), Lecture Notes in Mathematics Vol. 1812, Springer-Verlag, Berlin, 2003.

[41] C.B. Muratov, V.V. Osipov, Static spike autosolitons in the Gray-Scott model, J. Phys. A: Math. Gen. 33 (2000), 8893-8916.

[42] C.B. Muratov, V.V. Osipov, Stability of the static spike autosolitons in the Gray-Scott model, SIAM J. Appl. Math. 62 (2002), 1463-1487.

[43] J.D. Murray, Mathematical Biology II: Spatial Models and Biomedical Applications, Interdisciplinary Applied Mathematics Vol. 18, Springer, 2003.

[44] W.-M. Ni, Diffusion, cross-diffusion, and their spike-layer steady-states, Notices Am. Math. Soc. 45 (1998), 9-18.

[45] Y. Nishiura, Far-From-Equilibrium-Dynamics, Translations of Mathematical Monographs Vol. 209, AMS publications, Providence, Rhode Island, 2002.

[46] Y. Nishiura, Global structure of bifurcating solutions of some reaction-diffusion systems, SIAM J. Math. Anal. 13 (1982), 555-593.

[47] Y. Nishiura and H. Fujii, Stability of singularly perturbed solutions to systems of reaction-diffusion equations, SIAM J. Math. Anal. 18 (1987) 1726-1770.

[48] Y. Nishiura, T. Teramoto and K. Ueda, Scattering and separators in dissipative systems, Phys. Rev. E 67(5) (2003), 56210.

[49] Y. Nishiura and D. Ueyama, A skeleton structure of self-replicating dynamics, Physica D 130 (1999), $73-104$. 
[50] Y. Nishiura and D. Ueyama, Spatio-temporal chaos for the Gray-Scott model, Physica D 150 (2001), 137-162.

[51] Q. Ouyang and H. L. Swinney, Transition from a uniform state to hexagonal and striped Turing patterns, Nature 352 (1991), 610-612.

[52] Q. Ouyang and H. L. Swinney, Transition to chemical turbulence, Chaos 1 (1991), 411-420.

[53] K. J. Painter, P. K. Maini and H. G. Othmer, Stripe formation in juvenile pomacanthus explained by a generalized Turing mechanism with chemotaxis, Proc. Nat. Acad. Sci. USA, Dev. Biol. 96 (1999), 5549-5554.

[54] J.E. Pearson, Complex patterns in a simple system, Science 261 (1993), 189-192.

[55] J. E. Pearson and W. Horsthemke, Turing instabilities with nearly equal diffusion constants, J. Chem. Phys. 90 (1989), 1588-1599.

[56] J. Reynolds, J. Pearson and S. Ponce-Dawson, Dynamics of self-replicating patterns in reaction diffusion systems, Phys. Rev. Lett. 72 (1994), 2797-2800.

[57] J. Reynolds, J. Pearson and S. Ponce-Dawson, Dynamics of self-replicating spots in reaction-diffusion systems, Phys. Rev. E 56 (1997), 185-198.

[58] B. Sandstede and A. Scheel, Absolute inequalities of standing pulses, Nonlinearity 18 (2005), 331-378.

[59] J. Schnakenberg, Simple chemical reaction systems with limit cycle behaviour, J. Theoret. Biol. 81 (1979), 389-400.

[60] L. A. Segel and S. A. Levin, Appliations of nonlinear stability theory to the study of the effects of dispersion on predator-prey interactions. In: Selected Topics in Statistical Mechanics and Biophysics, Conference Proceedings no. 27., R. Piccirelli Ed., American Inst. Physics, New York, 1976.

[61] S. Sick, S. Reinker, J. Timmer and T. Schlake, WNT and DKK determine hair follicle spacing through a reactiondiffusion mechanism, Science 314 (2006), 1447-1450.

[62] W. Sun, M. J. Ward and R. Russell, The slow dynamics of two-spike solutions for the Gray-Scott and GiererMeinhardt systems: competition and oscillatory instabilities, SIAM J. Appl. Dyn. Sys. 4 (2005), 904-953.

[63] I. Takagi, Point-condensation for a reaction-diffusion system, J. Differential Equations 61 (1986), 208-249.

[64] A. M. Turing, The chemical basis of morphogenesis, Phil. Trans. Roy. Soc. Lond. B 237 (1952), 37-72.

[65] J. A. Vastano, J. E. Pearson, W. Horsthemke and H. L. Swinney, Chemical pattern formation with equal diffusion coefficients, Phys. Lett. A 124 (1987), 320-324.

[66] J. A. Vastano, J. E. Pearson, W. Horsthemke and H. L. Swinney, Turing patterns in an open reactor, J. Chem. Phys. 88 (1988), 6175-6181.

[67] M. J. Ward, Asymptotic methods for reaction- diffusion systems: past and present, Bull. Math. Biol. 68 (2006), 1151-1167.

[68] J. Wei, On single interior spike solutions of the Gierer-Meinhardt system: uniqueness and spectrum estimates, Europ. J. Appl. Math. 10 (1999), 353-378.

[69] J. Wei, Existence, stability and metastability of point condensation patterns generated by Gray-Scott system, Nonlinearity 12 (1999), 593-616.

[70] J. Wei, Pattern formations in two-dimensional Gray-Scott model: existence of single-spot solutions and their stability, Physica D 148 (2001), 20-48.

[71] M.J. Ward and J. Wei, The existence and stability of asymmetric spike patterns for the Schnakenberg model, Stud. Appl. Math. 109 (2002), 229-264.

[72] M.J. Ward and J. Wei, Hopf bifurcations and oscillatory instabilities of solutions for the one-dimensional GiererMeinhardt model, J. Nonlinear Sci. 13 (2003), 209-264.

[73] J. Wei and M. Winter, On the two-dimensional Gierer-Meinhardt system with strong coupling, SIAM J. Math. Anal. 30 (1999), 1241-1263.

[74] J. Wei and M. Winter, Spikes for the two-dimensional Gierer-Meinhardt system: The strong coupling case, $J$. Differential Equations 178 (2000), 478-518.

[75] J. Wei and M. Winter, Spikes for the two-dimensional Gierer-Meinhardt system: The weak coupling case, $J$. Nonlinear Science 11 (2001), 415-458.

[76] J. Wei and M. Winter, Existence and stability of multiple-spot solutions for the Gray-Scott model in $\mathbb{R}^{2}$ Phys. D 176 (2003), 147-180.

[77] J. Wei and M. Winter, Asymmetric spotty patterns for the Gray-Scott model in $R^{2}$, Stud. Appl. Math. 110 (2003), 63-102. 


\section{FIGURES}

Initial condition (same for all $D$ )
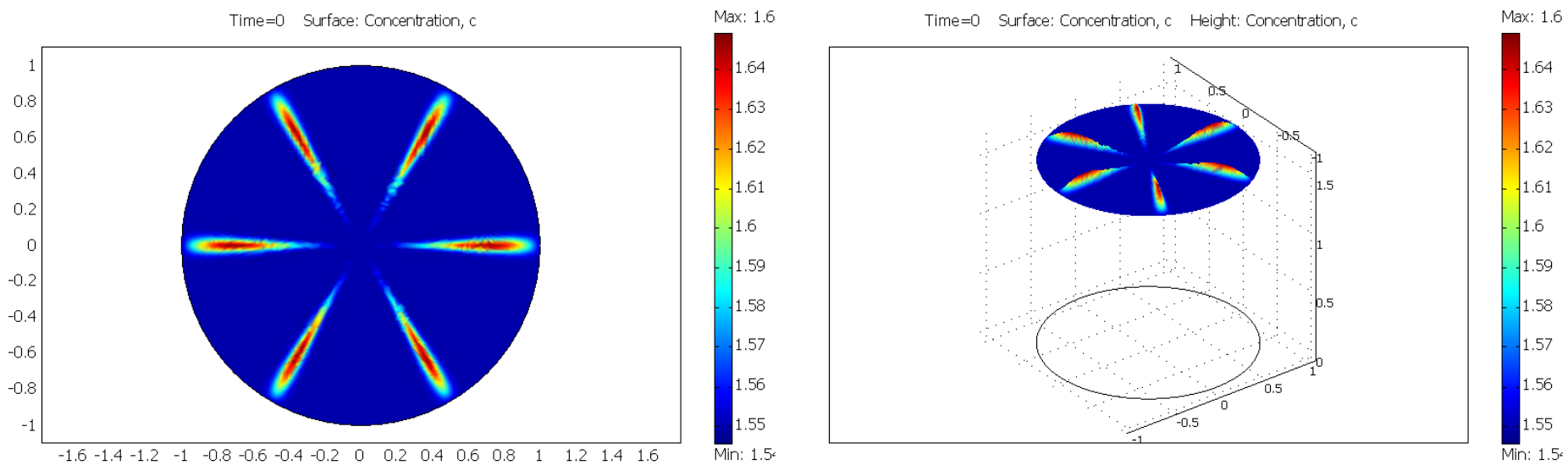

Figure 1. Initial condition (for $t=0$ ) on unit circle, for all $D$, given by $a(x, y)=1.55+0.1 \sin \pi\left(x^{2}+y^{2}\right) \sin ^{100} \varphi$, where $\varphi=\sin \left(\arcsin \left(\frac{x}{\sqrt{x^{2}+y^{2}}}\right)\right)$ is the angle with the $x$-axis. 
Final state for various $D$

$$
D=3.5
$$
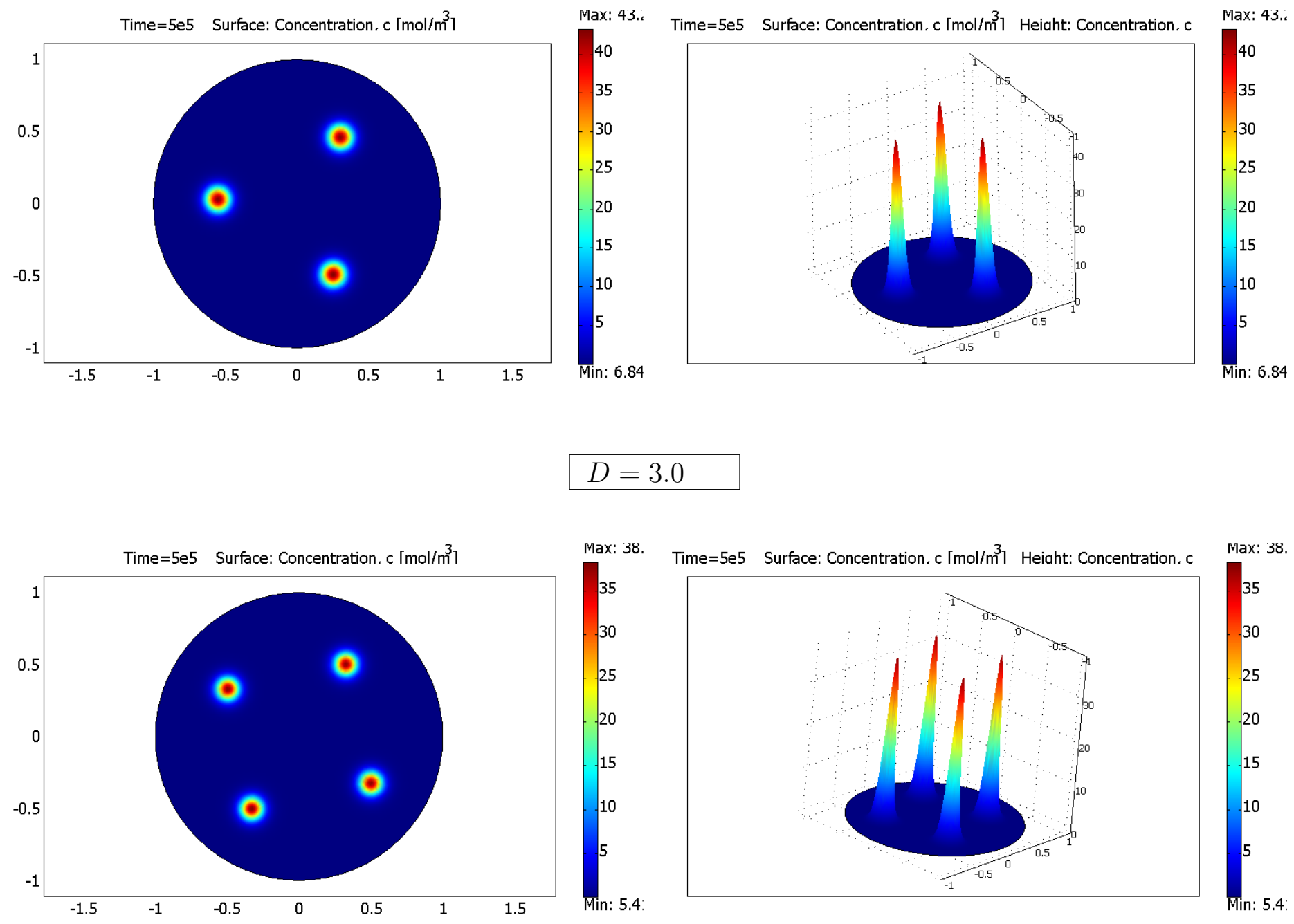

Max: 38.

$$
D=3.0
$$

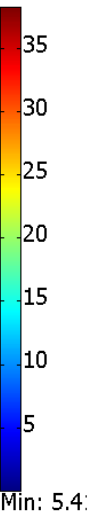

$$
D=2.5
$$
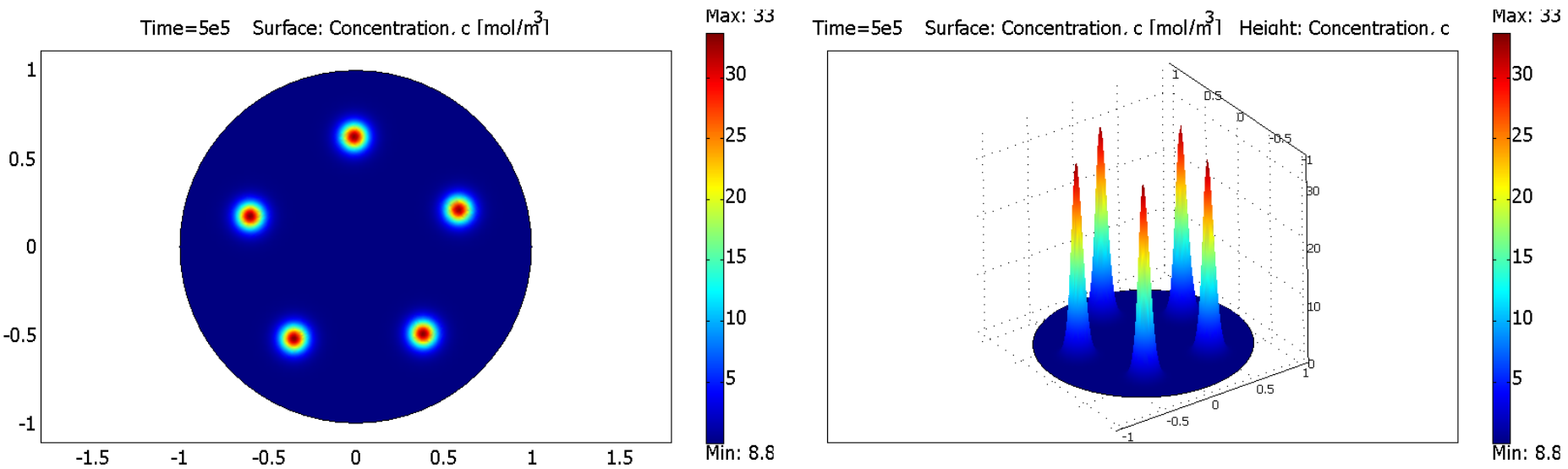


$$
D=2.0
$$
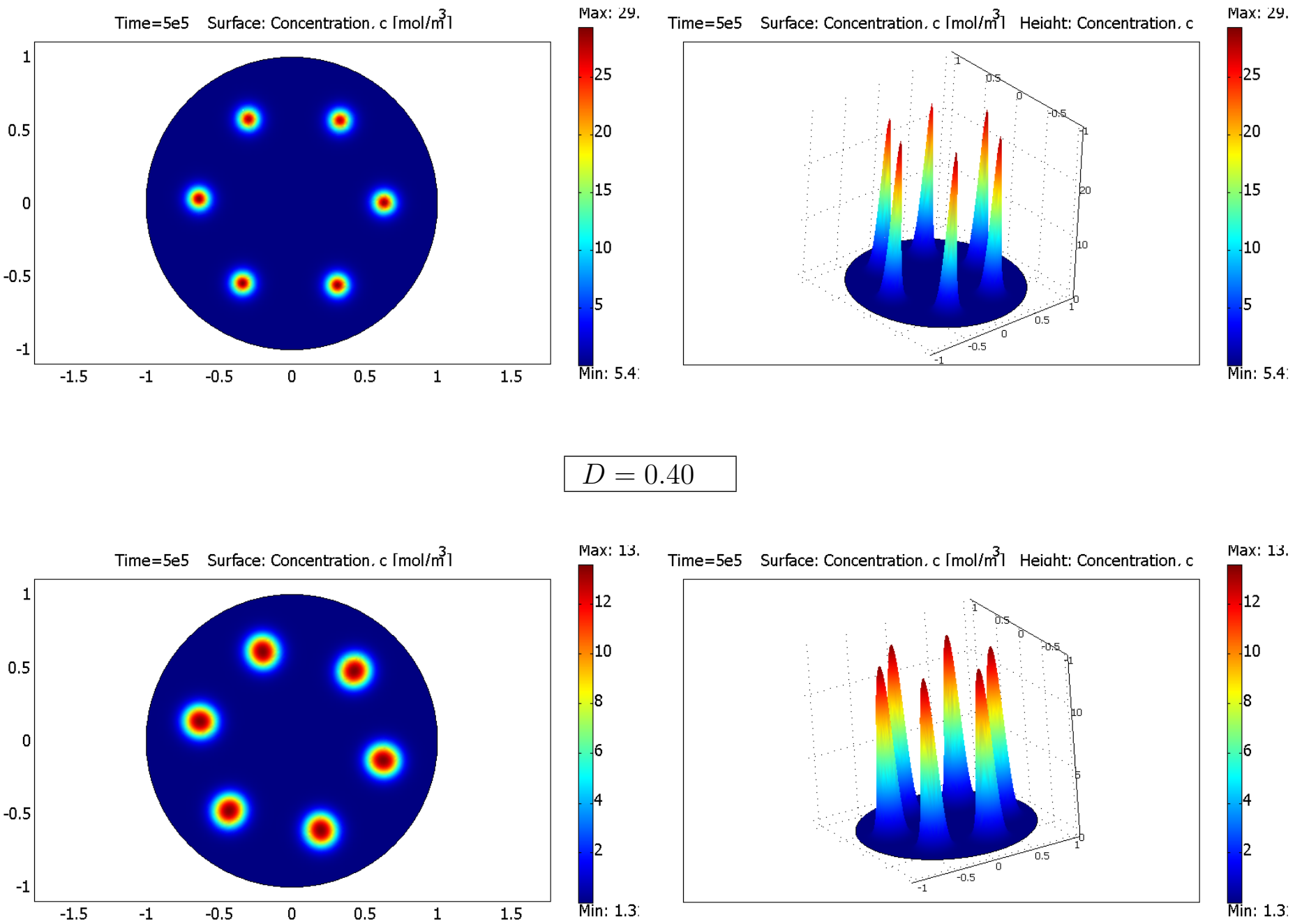

$$
D=0.30
$$
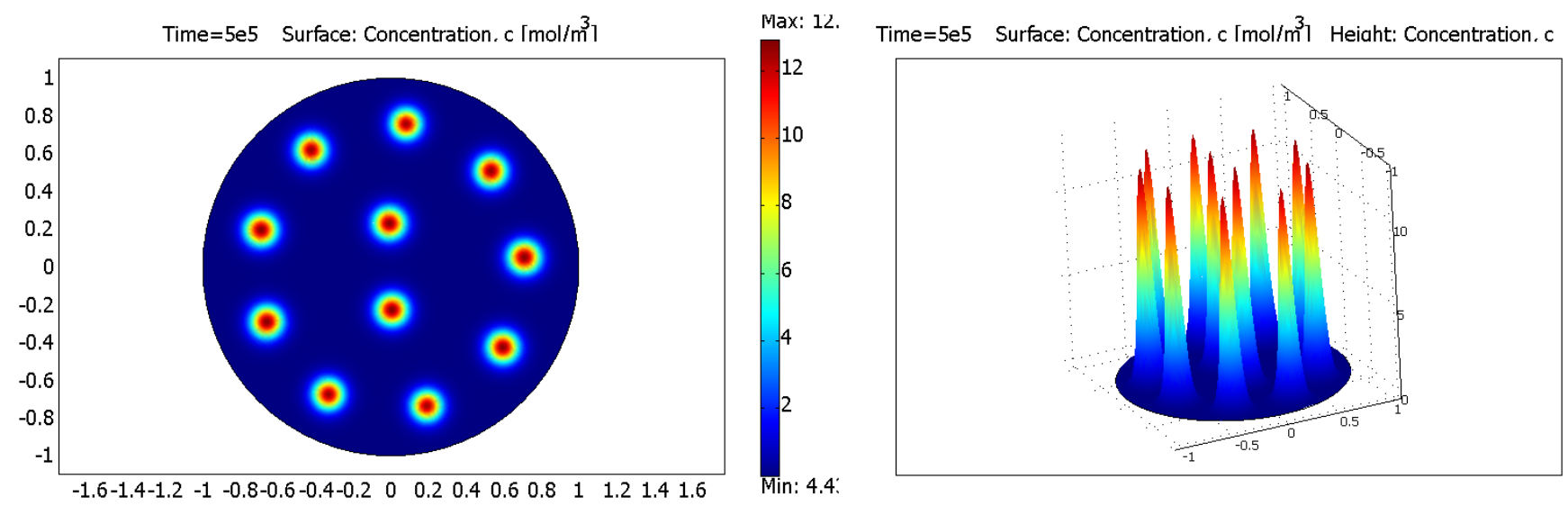

Max: 12.

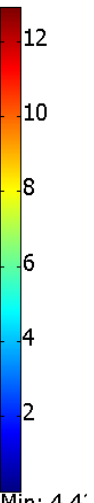

$D=0.10$ 
JUNCHENG WEI AND MATTHIAS WINTER
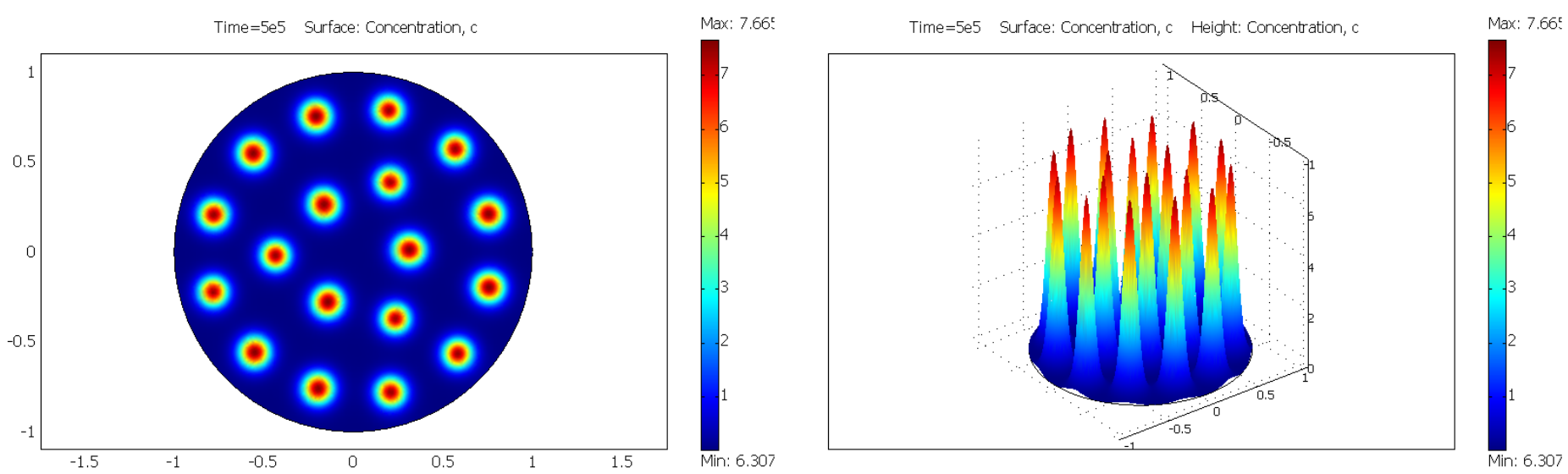


$$
D=0.03
$$
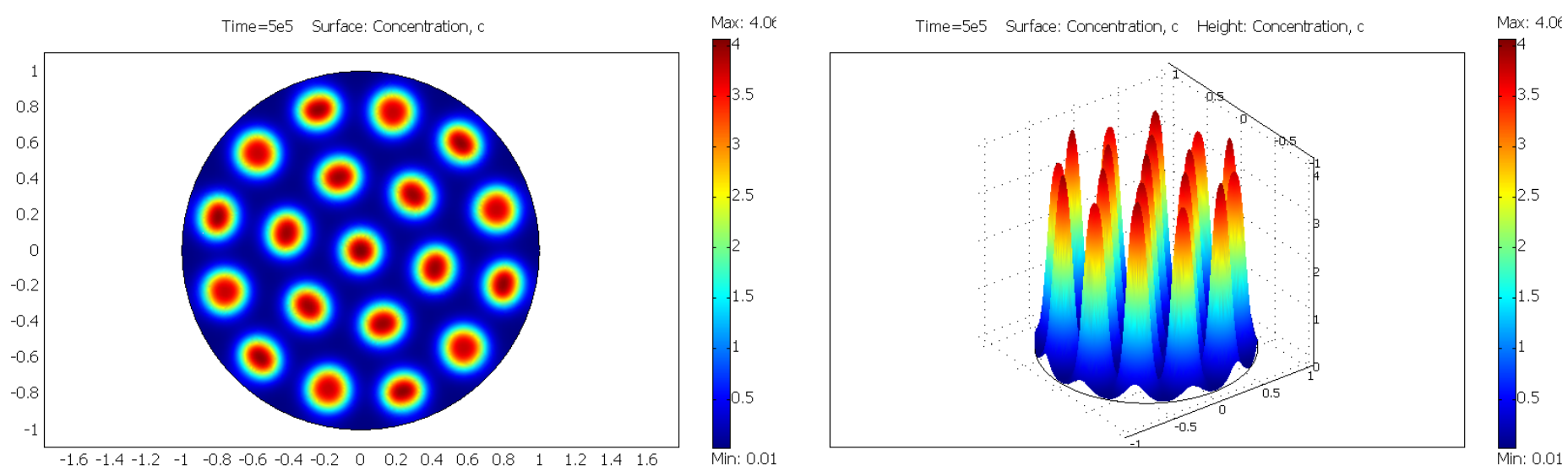

$$
D=0.01
$$
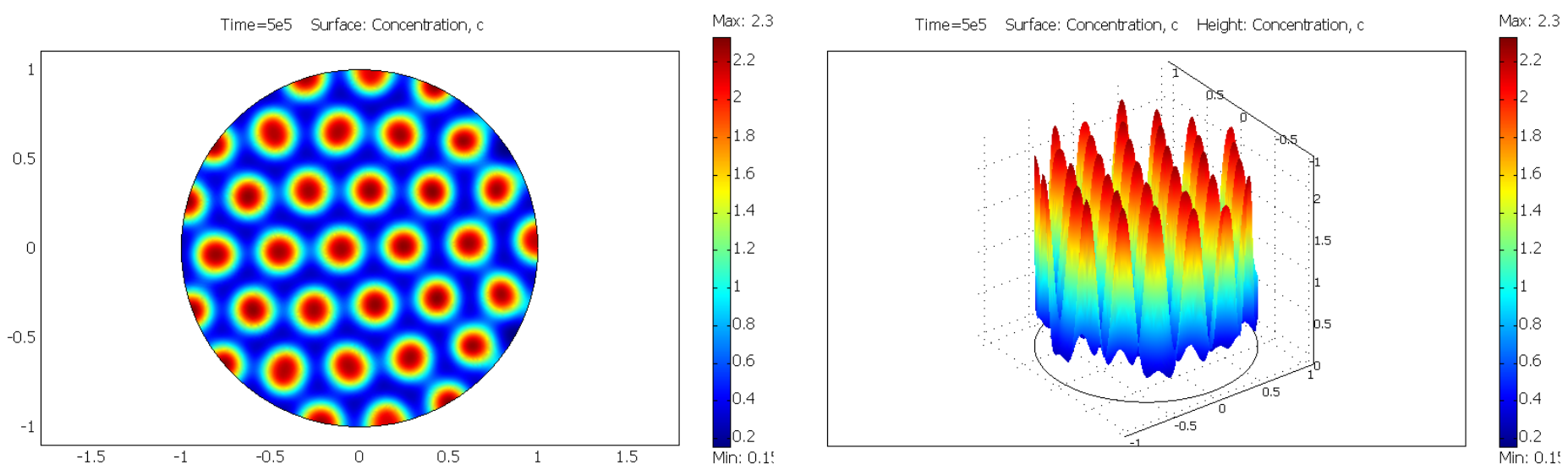

Figure 2. Plots for $D=3.5,3.0,2.5,2.0,0.40,0.30,0.10,0.03,0.01$. For decreasing $D$ more and more stable spots are possible.

Department of Mathematics, The Chinese University of Hong Kong, Shatin, Hong Kong E-mail address: wei@math.cuhk.edu.hk

Brunel University, Department of Mathematical Sciences, Uxbridge UB8 3PH, United Kingdom E-mail address: matthias.winter@brunel.ac.uk 